\title{
The generation of matter-antimatter asymmetries and hypermagnetic fields by the chiral vortical effect of transient fluctuations
}

\author{
S. Abbaslu ${ }^{1, a}$, S. Rostam Zadeh ${ }^{2, b}$, M. Mehraeen ${ }^{1,3, c}$, S. S. Gousheh ${ }^{1, d} \mathbb{C}_{\mathbb{C}}$ \\ ${ }^{1}$ Department of Physics, Shahid Beheshti University, Tehran, Iran \\ ${ }^{2}$ School of Particles and Accelerators, Institute for Research in Fundamental Sciences (IPM), P.O.Box 19395-5531, Tehran, Iran \\ ${ }^{3}$ Department of Physics, Case Western Reserve University, 10900 Euclid Avenue, Cleveland, OH 44106, USA
}

Received: 16 March 2021 / Accepted: 19 May 2021 / Published online: 6 June 2021

(C) The Author(s) 2021

\begin{abstract}
We study the contribution of the temperaturedependent chiral vortical effect to the generation and evolution of hypermagnetic fields and matter-antimatter asymmetries, in the symmetric phase of the early Universe, in the temperature range $100 \mathrm{GeV} \leq T \leq 10 \mathrm{TeV}$. Our most important result is that, due to the chiral vortical effect, small overlapping transient fluctuations in the vorticity field in the plasma and temperature of matter degrees of freedom can lead to the generation of strong hypermagnetic fields and matter-antimatter asymmetries, all starting from zero initial values. We show that, either an increase in the amplitudes of the fluctuations of vorticity or temperature, or a decrease in their widths, leads to the production of stronger hypermagnetic fields, and therefore, larger matter-antimatter asymmetries. We have the interesting result that fluctuating vorticity fields are more productive, by many orders of magnitude, as compared to vorticities that are constant in time.
\end{abstract}

\section{Introduction}

Anomalous transport effects play important roles in particle physics and cosmology, particularly in the early Universe [1]. One important effect of this kind is the so-called Chiral Vortical Effect (CVE), which refers to the generation of an electric current parallel to the vorticity field in the chiral plasma [2]. This effect was discovered by Vilenkin who showed that a neutrino current density can result from a rotating black hole [2]. He obtained the neutrino current density in the direction

\footnotetext{
a e-mail: s_abbasluo@sbu.ac.ir

be-mail: sh_rostamzadeh@ipm.ir

ce-mail: mxm1289@case.edu

de-mail: ss-gousheh@sbu.ac.ir (corresponding author)
}

of the rotation axis as

$J(0)=-\frac{\Omega}{12} T^{2}-\frac{\Omega}{4 \pi^{2}} \mu^{2}-\frac{\Omega^{3}}{48 \pi^{2}}$,

where $\Omega$ is the angular velocity, $\mu$ is the chiral chemical potential of the neutrino, and $T$ is its temperature. Thirty years after its discovery, the CVE appeared in the relativistic hydrodynamic equations as an interesting manifestation of anomalies in quantum field theory [3]. This effect has attracted much attention and has been investigated extensively in recent years, leading to a deeper understanding of the subject [4-10]. In particular, it has been established that in single species chiral plasma in the broken phase at high temperatures, the CVE shows up in the vector current as $\vec{J}_{\mathrm{cv}}=\frac{1}{4 \pi^{2}}\left(\mu_{R}^{2}-\mu_{L}^{2}\right) \vec{\Omega}$, and in the axial current as $\vec{J}_{\mathrm{cv}}^{5}=\left[\frac{T^{2}}{6}+\frac{1}{4 \pi^{2}}\left(\mu_{R}^{2}+\mu_{L}^{2}\right)\right] \vec{\Omega}$, where $\mu_{R}$ and $\mu_{L}$ are the right-handed and the left-handed chemical potentials of the species, respectively [2-10]. ${ }^{1}$ Interestingly, the term proportional to $T^{2}$ indicates that there can be an axial current, even if $\mu_{R}=\mu_{L}=0$.

In this study, we present the correct form of the chiral vortical current in the symmetric phase. Then, we show the prominent effects of the temperature-dependent part of this current in the symmetric phase of the early Universe close to the electroweak phase transition (EWPT). In particular, we show that even very small, but overlapping, transient fluctuations in the vorticity field and temperature of matter degrees of freedom can have important consequences, including the generation of hypermagnetic field in the absence of initial matter asymmetries. The vorticity fluctuations that we consider are about the zero background value, while the tempera-

\footnotetext{
1 There are additional contributions of $O(\mu / T)$, where $\mu$ is the chemical potential, which are significant at lower temperatures. See Appendix A for details.
} 
ture fluctuations are about the finite equilibrium temperature of the plasma. The most important role of the CVE in this context is to produce the magnetic fields, either through the chiralities, or through the temperature fluctuation, the latter of which is the main focus of this work. Henceforth, we shall refer to transient fluctuations, which we take to be in the form of short pulses, simply as fluctuations.

Another anomalous transport effect is the chiral magnetic effect (CME), which refers to the generation of an electric current parallel to the magnetic field in the imbalanced chiral plasma [11-14]. It is known that, in single species chiral plasma in the broken phase at high temperatures, the CME appears in the vector current as $\vec{J}_{\mathrm{cm}}=\frac{Q}{4 \pi^{2}}\left(\mu_{R}-\mu_{L}\right) \vec{B}$, and in the axial current as $\vec{J}_{\mathrm{cm}}^{5}=\frac{Q}{4 \pi^{2}}\left(\mu_{R}+\mu_{L}\right) \vec{B}$, where $Q$ is the electric charge $[10,11,15,16]$ of the species. ${ }^{1}$ Later, we will present the correct form of the chiral magnetic current, in the symmetric phase. The chiral magnetic current originating from the electroweak Abelian anomaly, and the chiral vortical current are both non-dissipative currents which can strongly affect the generation and the evolution of the magnetic fields and the matter-antimatter asymmetries in the early Universe [17-21].

Observations clearly show that our Universe is magnetized on all scales [22,23]. Various models have been proposed to explain the origin of these magnetic fields [20,24-30], among which the one relying on the electroweak Abelian anomaly has attracted much attention and has been considerably investigated [17-19,31]. There exists a relationship between the generation and the evolution of the hypermagnetic fields and the fermion number densities in this model, which is due to the chiral coupling of the hypercharge gauge fields to the fermions before the EWPT [27]. In case there is a preexisting asymmetry of the right-handed electrons, their number density is almost conserved far from the EWPT, i.e. $T>10 \mathrm{TeV}$, due to their tiny Yukawa coupling. For lower temperatures, this asymmetry can be converted to the hypermagnetic helicity according to the Abelian anomaly equation, $\partial_{\mu} j_{e_{R}}^{\mu} \sim \vec{E}_{Y} \cdot \vec{B}_{Y}[17-19,27,31,32]$. The anomaly equation shows that, in a reverse process, a strong helical hypermagnetic field can generate the matter-antimatter asymmetries in the Universe, as well [33-35].

Another challenge in particle physics and cosmology is the excess of matter over antimatter, with the measured baryon asymmetry of the Universe being of the order of $\eta_{\mathrm{B}} \sim 10^{-10}$ [36-39]. The three Sakharov conditions ${ }^{2}$ should be satisfied in any CPT invariant model used to explain this asymmetry from an initially symmetric Universe [40-43].

In previous studies based on the electroweak Abelian anomalous model, it has been assumed that there is either a significant amount of matter-antimatter asymmetries to

\footnotetext{
2 (i) Baryon number violation, (ii) $\mathrm{C}$ and $\mathrm{CP}$ violation, (iii) a departure from thermal equilibrium.
}

produce the hypermagnetic field, or a strong hypermagnetic field to produce the matter-antimatter asymmetries. The most important result of this study is that the matter-antimatter asymmetries and the hypermagnetic field can all be generated simultaneously from zero initial values, by considering the temperature-dependent CVE before the EWPT. To obtain this interesting result, we take into account the effects of the temperature-dependent term of the chiral vortical current on the evolution of the hypermagnetic fields and the matterantimatter asymmetries, by considering simultaneous small fluctuations, about the background values, in temperature of the right-handed electrons and the vorticity field, close to the EWPT. We also show that fluctuations in the vorticity field are much more productive than vorticity fields that are constant in time. To be more precise, sharp fluctuations yield results comparable to constant vorticities whose amplitudes are many orders of magnitude larger.

As mentioned above, an underlying assumption of this work is the presence of fluctuations in the plasma of the early Universe, containing all elementary particles and gauge fields. A general description of this plasma in the context of hydrodynamics, as an effective field theory, has been presented $[44,45]$. In such a physical system, hydrodynamic variables naturally fluctuate around their statistical averages. Such stochastic and frequent fluctuations are a commonplace in any plasma, including that of the early Universe. These fluctuations, which may stem from a sum of weakly-correlated random local events, can occur for physical observables such as the number density, velocity, and temperature [44,46-48]. Even though the gauge interactions are strong and their rates are fast in this epoch, the extreme temperatures of the primordial plasma imply that fluctuations could still occur for all species. Moreover, owing to the different Yukawa and gauge couplings, and in particular the chiral nature of the electroweak sector, different chiral fermions could, in principle, experience different fluctuations. All that is required here is that the fluctuations for at least one of the species be non-identical to the rest, at least once.

Another justification for our hypothesis is the following. Realistic fluctuations of density, temperature and vorticity are usually local in space and time. Meanwhile, in a multicomponent plasma local and independent density fluctuations can occur for different matter components. The concurrent occurrence of density, temperature and vorticity fluctuations leads to the possibility of occurrence of non-identical fluctuations for different species of particles. To be concrete, consider a local density fluctuation leading to a local excess of a particle species, e.g. $e_{R}$, in the central region and rarefaction in the surrounding region, followed immediately by a temperature fluctuation which increases the temperature of the central region. The concurrent occurrence of these two fluctuations leads to a temporary local, and to a much lesser degree global average, increase of temperature of that parti- 
cle species relative to quasi-equilibrium temperature of the plasma. All that is required now is an overlapping vorticity fluctuation to have the necessary conditions for our model. In this work, we make the simplifying assumption, as is usually done, of considering these fluctuations to be global rather than local. It should be noted that the amplitude and duration of these fluctuations are usually small enough such that the equilibrium energy density, pressure and entropy of the system can be defined. Nevertheless, as we shall show, even very small and brief fluctuations can trigger the mechanism that we propose here and affect significantly the dynamical evolution of the system, as is formulated within the equations of anomalous magnetohydrodynamics (AMHD).

This paper is organized as follows: In Sect. 2, the anomalous magnetohydrodynamics equations and the evolution equations for the matter-antimatter asymmetries are derived in the expanding Universe. In Sect. 3, the set of coupled differential equations are solved numerically. In Sect. 4, the results are summarized and the conclusion is presented.

\section{Evolution equations}

\subsection{Anomalous magnetohydrodynamics equations}

In this section, the anomalous magnetohydrodynamics (AMHD) equations are obtained in the Landau-Lifshitz frame in the symmetric phase of the expanding Universe. Taking the CVE and the CME into account, the Maxwell's equations for the hypercharge-neutral plasma in the expanding Universe are given as $[3,20,49-56]$

$$
\begin{aligned}
& \frac{1}{R} \vec{\nabla} \cdot \vec{E}_{Y}=0, \quad \frac{1}{R} \vec{\nabla} \cdot \vec{B}_{Y}=0, \\
& \frac{1}{R} \vec{\nabla} \times \vec{E}_{Y}+\left(\frac{\partial \vec{B}_{Y}}{\partial t}+2 H \vec{B}_{Y}\right)=0, \\
& \frac{1}{R} \vec{\nabla} \times \vec{B}_{Y}-\left(\frac{\partial \vec{E}_{Y}}{\partial t}+2 H \vec{E}_{Y}\right)=\vec{J} \\
& \quad=\vec{J}_{\mathrm{Ohm}}+\vec{J}_{\mathrm{cv}}+\vec{J}_{\mathrm{cm}}, \\
& \vec{J}_{\mathrm{Ohm}}=\sigma\left(\vec{E}_{Y}+\vec{v} \times \vec{B}_{Y}\right), \\
& \vec{J}_{\mathrm{cv}}=c_{\mathrm{v}} \vec{\omega} \\
& \vec{J}_{\mathrm{cm}}=c_{\mathrm{B}} \vec{B}_{Y}
\end{aligned}
$$

where $R$ is the scale factor, $H=\dot{R} / R$ is the Hubble parameter, $\sigma$ is the electrical hyperconductivity of the plasma, and $\vec{v}$ and $\vec{\omega}=\frac{1}{R} \vec{\nabla} \times \vec{v}$ are the bulk velocity and vorticity of the plasma, respectively. Furthermore, the chiral vorticity and helicity coefficients $c_{\mathrm{V}}$ and $c_{\mathrm{B}}$ are as follows (see
Appendix A), ${ }^{3}$

$$
\begin{aligned}
c_{\mathrm{V}}(t)= & \sum_{i=1}^{n_{G}}\left[\frac { g ^ { \prime } } { 4 8 } \left(-Y_{R} T_{R_{i}}^{2}+Y_{L} T_{L_{i}}^{2} N_{w}\right.\right. \\
& \left.-Y_{d_{R}} T_{d_{R_{i}}}^{2} N_{c}-Y_{u_{R}} T_{u_{R_{i}}}^{2} N_{c}+Y_{Q} T_{Q_{i}}^{2} N_{c} N_{w}\right) \\
& +\frac{g^{\prime}}{16 \pi^{2}}\left(-Y_{R} \mu_{R_{i}}^{2}+Y_{L} \mu_{L_{i}}^{2} N_{w}-Y_{d_{R}} \mu_{d_{R_{i}}}^{2} N_{c}\right. \\
& \left.\left.-Y_{u_{R}} \mu_{u_{R_{i}}}^{2} N_{c}+Y_{Q} \mu_{Q_{i}}^{2} N_{c} N_{w}\right)\right], \\
c_{\mathrm{B}}(t)= & -\frac{g^{\prime 2}}{8 \pi^{2}} \sum_{i=1}^{n_{G}}\left[-\left(\frac{1}{2}\right) Y_{R}^{2} \mu_{R_{i}}-\left(\frac{-1}{2}\right) Y_{L}^{2} \mu_{L_{i}} N_{w}\right. \\
& -\left(\frac{1}{2}\right) Y_{d_{R}}^{2} \mu_{d_{R_{i}}} N_{c}-\left(\frac{1}{2}\right) Y_{u_{R}}^{2} \mu_{u_{R_{i}}} N_{c} \\
& \left.-\left(\frac{-1}{2}\right) Y_{Q}^{2} \mu_{Q_{i}} N_{c} N_{w}\right],
\end{aligned}
$$

where $n_{G}$ is the number of generations, and $N_{c}=3$ and $N_{w}=2$ are the ranks of the non-Abelian SU(3) and SU(2) gauge groups, respectively. Moreover, $\mu_{L_{i}}\left(\mu_{R_{i}}\right), \mu_{Q_{i}}$, and $\mu_{u_{R i}}\left(\mu_{d_{R i}}\right)$ are the common chemical potentials of lefthanded (right-handed) leptons, left-handed quarks with different colors, and up (down) right-handed quarks with different colors, respectively. Furthermore, ' $i$ ' is the generation index, and the relevant hypercharges are

$$
\begin{aligned}
& Y_{L}=-1, \quad Y_{R}=-2, \\
& Y_{Q}=\frac{1}{3}, \quad Y_{u_{R}}=\frac{4}{3}, \quad Y_{d_{R}}=-\frac{2}{3} .
\end{aligned}
$$

After substituting the hypercharges in Eqs. (2.7) and (2.8), we obtain

$$
\begin{aligned}
c_{\mathrm{v}}(t)= & \sum_{i=1}^{n_{G}}\left[\frac{g^{\prime}}{24}\left(T_{R_{i}}^{2}-T_{L_{i}}^{2}+T_{d_{R_{i}}}^{2}-2 T_{u_{R_{i}}}^{2}+T_{Q_{i}}^{2}\right)\right. \\
& \left.+\frac{g^{\prime}}{8 \pi^{2}}\left(\mu_{R_{i}}^{2}-\mu_{L_{i}}^{2}+\mu_{d_{R_{i}}}^{2}-2 \mu_{u_{R_{i}}}^{2}+\mu_{Q_{i}}^{2}\right)\right] \\
c_{\mathrm{B}}(t)= & \frac{-g^{\prime 2}}{8 \pi^{2}} \sum_{i=1}^{n_{G}}\left[-2 \mu_{R_{i}}+\mu_{L_{i}}-\frac{2}{3} \mu_{d_{R_{i}}}-\frac{8}{3} \mu_{u_{R_{i}}}+\frac{1}{3} \mu_{Q_{i}}\right] .
\end{aligned}
$$

Let us make the same assumptions as in our previous studies, and simplify $c_{\mathrm{V}}$ and $c_{\mathrm{B}}$ accordingly $[18,20]$. We assume that all quark Yukawa processes are in equilibrium and, because of the flavor mixing in the quark sector, all up or down quarks belonging to different generations with distinct handedness have the same chemical potential $[18,57,58]$. For

\footnotetext{
3 The temperature-independent parts of these coefficients were presented in [20]. There are additional terms of $O(\mu / T)$ which, as we shall show explicitly in Sect. 3, are negligible within the initial conditions and results of our model (see Appendix A).
} 
simplicity, we also assume that the Higgs asymmetry is zero and obtain $[18,59]$

$\mu_{u_{R}}=\mu_{d_{R}}=\mu_{Q}$.

Furthermore, we assume that only the contributions of the baryonic and the first-generation leptonic chemical potentials to $c_{\mathrm{V}}$ and $c_{\mathrm{B}}$ are significant. As for the temperature fluctuations, it suffices to consider fluctuations in only one of the matter components, which we take to be $e_{R}$. Using Eq. (2.12) and the aforementioned assumptions, we simplify Eqs. (2.10) and (2.11) to obtain

$c_{\mathrm{V}}(t)=\frac{g^{\prime}}{24}\left(\Delta T^{2}\right)+\frac{g^{\prime}}{8 \pi^{2}}\left(\mu_{e_{R}}^{2}-\mu_{e_{L}}^{2}\right)$,

$c_{\mathrm{B}}(t)=-\frac{g^{\prime 2}}{8 \pi^{2}}\left(-2 \mu_{e_{R}}+\mu_{e_{L}}-\frac{3}{4} \mu_{\mathrm{B}}\right)$,

where $\mu_{\mathrm{B}}=12 \mu_{Q}$, and $\Delta T^{2}=T_{e_{R}}^{2}-T^{2}$ is the temperature fluctuation, and $T$ is the equilibrium temperature of the thermal bath, which includes all other components of the plasma. We set $\Delta T^{2}=T^{2} \beta[x(T)]$, where $\beta[x(T)]$ is an arbitrary profile function to be specified later, and $x(T)=t(T) / t_{\mathrm{EW}}=\left(T_{\mathrm{EW}} / T\right)^{2}$ is given by the Friedmann law.

In the Landau-Lifshitz frame, the continuity and NavierStokes equations are given as follows [20,51-53]

$$
\begin{aligned}
& \frac{\partial \rho}{\partial t}+\frac{1}{R} \vec{\nabla} \cdot[(\rho+p) \vec{v}]+3 H(\rho+p)=0, \\
& {\left[\frac{\partial}{\partial t}+\frac{1}{R}(\vec{v} \cdot \vec{\nabla})+H\right] \vec{v}+\frac{\vec{v}}{\rho+p} \frac{\partial p}{\partial t}} \\
& \quad=-\frac{1}{R} \frac{\vec{\nabla} p}{\rho+p}+\frac{\vec{J} \times \vec{B}_{Y}}{\rho+p}+\frac{v}{R^{2}}\left[\nabla^{2} \vec{v}+\frac{1}{3} \vec{\nabla}(\vec{\nabla} \cdot \vec{v})\right],
\end{aligned}
$$

where $v$ is the kinematic viscosity, and $\rho$ and $p$ are the energy density and the pressure of the plasma, respectively. Combining the fluid incompressibility condition in the lab frame, $\partial_{t} \rho+3 H(\rho+p)=0$ or equivalently $H \vec{v}+\vec{v} \partial_{t} p /(\rho+p)=0$, with Eq. (2.15) leads to the condition $\vec{\nabla} \cdot \vec{v}=0[20,53,60]$.

In the following, we choose a simple monochromatic Chern-Simons configuration for the hypermagnetic field $\vec{B}_{Y}=(1 / R) \vec{\nabla} \times \vec{A}_{Y}$, and the velocity field $\vec{v}=(1 / R) \vec{\nabla} \times \vec{S}$ $[18,20]$. To do this, we choose $\vec{A}_{Y}=\gamma(t)(\cos k z, \sin k z, 0)$, and $\vec{S}=r(t)(\cos k z, \sin k z, 0)$, for their corresponding vector potentials [61-63]. Note that we have chosen a fully helical form with the negative helicity for both, the reason for which will be stated later. Let us now obtain the evolution equation for the velocity field. Neglecting the displacement current in the lab frame in Eq. (2.3), the total current becomes $\vec{J}=(1 / R) \vec{\nabla} \times \vec{B}_{Y}$, and as a result, $\vec{J} \times \vec{B}_{Y}$ vanishes in Eq. (2.16). Then, using $\vec{\nabla} \cdot \vec{v}=0$ and $H \vec{v}+\vec{v} \partial_{t} p /(\rho+p)=0$, as stated earlier, and neglecting the gradient terms in Eq. (2.16), the evolution equation for the velocity field becomes ${ }^{4}$

$\frac{\partial \vec{v}}{\partial t}=-v k^{\prime 2} \vec{v}$

where the kinematic viscosity $v \simeq 1 /\left(5 \alpha_{Y}^{2} T\right)[65,66]$. Neglecting the displacement current in the lab frame and using the aforementioned configurations, the hyperelectric field and the evolution equation for the hypermagnetic field are obtained, as follows:

$$
\begin{aligned}
& \vec{E}_{Y}=-\frac{k^{\prime}}{\sigma} \vec{B}_{Y}+\frac{c_{\mathrm{V}}}{\sigma} k^{\prime} \vec{v}-\frac{c_{\mathrm{B}}}{\sigma} \vec{B}_{Y} \\
& \frac{d B_{Y}(t)}{d t}=\left[-\frac{1}{t}-\frac{k^{\prime 2}}{\sigma}-\frac{c_{\mathrm{B}} k^{\prime}}{\sigma}\right] B_{Y}(t)+\frac{c_{\mathrm{V}}}{\sigma} k^{\prime 2}\left\langle\vec{v}(t) \cdot \hat{B}_{Y}(t)\right\rangle,
\end{aligned}
$$

where $\vec{\omega}=-k^{\prime} \vec{v}, \sigma=100 T$, angle brackets denote spatial average, and $k^{\prime}=k / R=k T$. The latter shows the increase of the hypermagnetic length scale, due to the expansion of the Universe. Note that with the choice of vector potentials for $\vec{v}(t)$ and $\vec{B}_{Y}(t)$, the advection term $\vec{v} \times \vec{B}_{Y}$ has been set to zero in the above, and we have the following simplification: $\left\langle\vec{v}(t) . \hat{B}_{Y}(t)\right\rangle \rightarrow v(t)$. In the next subsection we obtain the evolution equations for the matter-antimatter asymmetries.

\subsection{Evolution equations for the matter-antimatter asymmetries}

Before the EWPT, the gauge fields of $U_{Y}(1)$ couple to the fermions chirally, in contrast to those of $U_{\mathrm{em}}(1)$ in the broken phase, leading to the non-conservation of the matter currents. This shows up in the Abelian anomaly equations [67], which, for the first-generation leptons, are

$$
\begin{aligned}
& \nabla_{\mu} j_{e_{R}}^{\mu}=-\frac{1}{4}\left(Y_{R}^{2}\right) \frac{g^{\prime 2}}{16 \pi^{2}} Y_{\mu \nu} \tilde{Y}^{\mu \nu}=\frac{g^{\prime 2}}{4 \pi^{2}} \vec{E}_{Y} \cdot \vec{B}_{Y}, \\
& \nabla_{\mu} j_{e_{L}}^{\mu}=\frac{1}{4}\left(Y_{L}^{2}\right) \frac{g^{\prime 2}}{16 \pi^{2}} Y_{\mu \nu} \tilde{Y}^{\mu \nu}=-\frac{g^{\prime 2}}{16 \pi^{2}} \vec{E}_{Y} \cdot \vec{B}_{Y},
\end{aligned}
$$

where $\nabla_{\mu}$ is the covariant derivative with respect to FriedmannRobertson-Walker (FRW) metric $d s^{2}=d t^{2}-R^{2}(t) \delta_{i j} d x^{i} d x^{j}$, $t$ is the physical time, and $x^{i}$ s are the comoving coordinates. Integrating the above equations over all space and consider-

\footnotetext{
${ }^{4}$ The term $(\vec{v} \cdot \vec{\nabla}) \vec{v}$ is neglected because of being next to leading order. Furthermore, the magnetic pressure $B^{2} / 8 \pi$ is negligible compared to the fluid (radiation) pressure $p$ which is homogeneous and isotropic. Indeed, the maximum value of their ratio at the onset of the EWPT is $B^{2} / 8 \pi p \approx 10^{-7} \ll 1$. Therefore, to a good approximation, the homogeneity and isotropy conditions remain valid and the pressure variations in the fluid $\vec{\nabla} p$ can be neglected [64].
} 
ing the perturbative chirality flip reactions for the leptons, we obtain (see Refs. [17, 18,20,68] and Appendix B for details),

$$
\begin{aligned}
& \frac{d \eta_{e_{R}}}{d t}= \frac{g^{\prime 2}}{4 \pi^{2} s}\left\langle\vec{E}_{Y} \cdot \vec{B}_{Y}\right\rangle+\left(\frac{\Gamma_{0}}{t_{E W}}\right)\left(\frac{1-x}{\sqrt{x}}\right)\left(\eta_{e_{L}}-\eta_{e_{R}}\right) \\
&-\frac{d}{d t}\left[\frac{g^{\prime} \mu_{e_{R}}}{4 \pi^{2} s}\left\langle\vec{v} \cdot \vec{B}_{Y}\right\rangle\right] \\
&-\frac{d}{d t}\left[\left(\frac{\mu_{e_{R}}^{2}}{8 \pi^{2} s}+\frac{T^{2}}{24 s}\right)\langle\vec{v} \cdot \vec{\omega}\rangle\right]+\frac{d}{d t}\left[\frac{2 \sigma_{e_{R}}}{g^{\prime} Y_{R} s}\left\langle\vec{v} \cdot \vec{E}_{Y}\right\rangle\right] \\
& \frac{d \eta_{v_{e}^{L}}}{d t}=\frac{d \eta_{e_{L}}}{d t}=-\frac{g^{2}}{16 \pi^{2} s}\left\langle\vec{E}_{Y} \cdot \vec{B}_{Y}\right\rangle \\
&+\left(\frac{\Gamma_{0}}{2 t_{E W}}\right)\left(\frac{1-x}{\sqrt{x}}\right)\left(\eta_{e_{R}}-\eta_{e_{L}}\right) \\
&+\frac{d}{d t}\left[\frac{g^{\prime} \mu_{e_{L}}}{8 \pi^{2} s}\left\langle\vec{v} \cdot \vec{B}_{Y}\right\rangle\right] \\
&+\frac{d}{d t}\left[\left(\frac{\mu_{e_{L}}^{2}}{8 \pi^{2} s}+\frac{T^{2}}{24 s}\right)\langle\vec{v} \cdot \vec{\omega}\rangle\right]+\frac{d}{d t}\left[\frac{2 \sigma_{e_{L}}}{g^{\prime} Y_{L} s}\left\langle\vec{v} \cdot \vec{E}_{Y}\right\rangle\right] .
\end{aligned}
$$

In the equations above, $\eta_{f}=\left(n_{f} / s\right)$ with $f=e_{R}, e_{L}, v_{e}^{L}$ is the fermion asymmetry and $n_{f}$ is the charge density of the $f$ th species of fermion, $s=2 \pi^{2} g^{*} T^{3} / 45$ is the entropy density and $g^{*}=106.75$ is the effective number of relativistic degrees of freedom, $x=\left(t / t_{\mathrm{EW}}\right)=\left(T_{\mathrm{EW}} / T\right)^{2}$ is given by the Friedmann law, $\Gamma_{0}=121, t_{\mathrm{EW}}=\left(M_{0} / 2 T_{\mathrm{EW}}^{2}\right)$, and $M_{0}=\left(M_{\mathrm{Pl}} / 1.66 \sqrt{g^{*}}\right)$, where $M_{\mathrm{Pl}}$ is the Plank mass. Furthermore, the term $\frac{\Gamma_{0}}{t_{\mathrm{EW}}}\left(\frac{1-x}{\sqrt{x}}\right)$ appearing in the equations is the chirality flip rate of the right-handed electrons. In a similar manner, the evolution equation for the baryon asymmetry can also be obtained as (see Appendix B)

$\frac{d \eta_{\mathrm{B}}}{d t}=\frac{3 g^{2}}{8 \pi^{2} s}\left\langle\vec{E}_{Y} \cdot \vec{B}_{Y}\right\rangle+\frac{d}{d t}\left[\frac{2}{s g^{\prime}}\left(\frac{\sigma_{d_{R}}}{Y_{d_{R}}}+\frac{\sigma_{u_{R}}}{Y_{u_{R}}}+2 \frac{\sigma_{Q}}{Y_{Q}}\right)\left\langle\vec{v} \cdot \vec{E}_{Y}\right\rangle\right]$.

Using $\mu_{f}=\left(6 s / T^{2}\right) \eta_{f}$ with Eq. (2.18) we obtain

$$
\begin{aligned}
& \left\langle\vec{E}_{Y} \cdot \vec{B}_{Y}\right\rangle=\frac{B_{Y}^{2}(t)}{100}\left[-\frac{k^{\prime}}{T}-\frac{6 s g^{\prime 2}}{4 \pi^{2} T^{3}}\left(\eta_{e_{R}}-\frac{\eta_{e_{L}}}{2}+\frac{3}{8} \eta_{\mathrm{B}}\right)\right] \\
& +\left[\frac{g^{\prime}}{24} \beta[x(T)]+\frac{36 s^{2} g^{\prime}}{8 \pi^{2} T^{6}}\left(\eta_{e_{R}}^{2}-\eta_{e_{L}}^{2}\right)\right] \frac{k^{\prime} T}{100}\left\langle\vec{v}(t) \cdot \vec{B}_{Y}(t)\right\rangle .
\end{aligned}
$$

With the helical configurations chosen, $\left\langle\vec{v}(t) \cdot \vec{B}_{Y}(t)\right\rangle \rightarrow$ $v(t) B_{Y}(t)$. Using 1 Gauss $\simeq 2 \times 10^{-20} \mathrm{GeV}^{2}$, and setting the kinematic viscosity $v$ to zero for simplicity, we obtain the complete set of evolution equations for the matter-antimatter asymmetries and the amplitudes of the hypermagnetic and velocity fields as

$$
\begin{aligned}
\frac{d \eta_{e_{R}}}{d x}= & \frac{1}{\lambda_{R}(x)}\left[\Lambda_{R}(x)+\left[-C_{1}-C_{2} \eta_{T}(x)\right]\left(\frac{B_{Y}(x)}{10^{20} G}\right)^{2} x^{3 / 2}\right. \\
& \left.+\left[C_{3} \beta(x)+C_{4} \Delta \eta^{2}(x)\right] v(x)\left(\frac{B_{Y}(x)}{10^{20} G}\right) \sqrt{x}\right] \\
& -\Gamma_{0} \frac{1-x}{\sqrt{x}}\left[\eta_{e_{R}}(x)-\eta_{e_{L}}(x)\right],
\end{aligned}
$$

$$
\begin{aligned}
& \frac{d \eta_{e_{L}}}{d x}=\frac{1}{\lambda_{L}(x)}\left[\Lambda_{L}(x)-\frac{1}{4}\left[-C_{1}-C_{2} \eta_{T}(x)\right]\left(\frac{B_{Y}(x)}{10^{20} G}\right)^{2} x^{3 / 2}\right. \\
& \left.-\frac{1}{4}\left[C_{3} \beta(x)+C_{4} \Delta \eta^{2}(x)\right] v(x)\left(\frac{B_{Y}(x)}{10^{20} G}\right) \sqrt{x}\right] \\
& +\Gamma_{0} \frac{1-x}{2 \sqrt{x}}\left[\eta_{e_{R}}(x)-\eta_{e_{L}}(x)\right], \\
& \frac{d B_{Y}}{d x}=\frac{1}{\sqrt{x}}\left[-C_{5}-C_{6} \eta_{T}(x)\right] B_{Y}(x) \\
& -\frac{1}{x} B_{Y}(x)+\left[C_{7} \beta(x)+C_{8} \Delta \eta^{2}(x)\right] \frac{v(x)}{x^{3 / 2}}, \\
& \frac{d \eta_{\mathrm{B}}}{d x}=\frac{3}{2}\left[-C_{1}-C_{2} \eta_{T}(x)\left(\frac{B_{Y}(x)}{10^{20} G}\right)^{2} x^{3 / 2}\right. \\
& \left.+\left[C_{3} \beta(x)+C_{4} \Delta \eta^{2}(x)\right] v(x)\left(\frac{B_{Y}(x)}{10^{20} G}\right) \sqrt{x}\right] \\
& +\frac{81 x}{100 M g^{\prime} 10^{20} G}\left\langle\vec{E}_{Y}(x) . \partial_{x} \vec{v}(x)\right\rangle \\
& \Delta \eta^{2}(x)=\eta_{e_{R}}^{2}(x)-\eta_{e_{L}}^{2}(x), \\
& \eta_{T}(x)=\eta_{e_{R}}(x)-\frac{\eta_{e_{L}}(x)}{2}+\frac{3}{8} \eta_{\mathrm{B}(x)}, \\
& \lambda_{R}(x)=1-\frac{6 g^{\prime} Y_{R}}{8 \pi^{2}} \frac{\left\langle\vec{v}(x) \cdot \vec{B}_{Y}(x)\right\rangle}{10^{20} G} \frac{x}{5000} \\
& -\frac{36 M}{4 \pi^{2}} \eta_{e_{R}}(x) k v^{2}(x), \\
& \lambda_{L}(x)=1+\frac{6 g^{\prime} Y_{L}}{8 \pi^{2}} \frac{\left\langle\vec{v}(x) \cdot \vec{B}_{Y}(x)\right\rangle}{10^{20} G} \frac{x}{5000} \\
& +\frac{36 M}{4 \pi^{2}} \eta_{e_{L}}(x) k v^{2}(x), \\
& \Lambda_{R}(x)=\frac{6 g^{\prime} Y_{R}}{8 \pi^{2}} \frac{x}{5000} \frac{\eta_{e_{R}}(x)}{10^{20} G}\left[\left\langle\vec{v}(x) \cdot \partial_{x} \vec{B}_{Y}(x)\right\rangle\right. \\
& \left.+\left\langle\frac{\vec{v}(x) \cdot \vec{B}_{Y}(x)}{x}\right\rangle+\left\langle\vec{B}_{Y}(x) \cdot \partial_{x} \vec{v}(x)\right\rangle\right] \\
& +\left[\frac{36 M}{4 \pi^{2}} \eta_{e_{R}}^{2}(x)+\frac{1}{12 M}\right] k \vec{v}(x) \cdot \partial_{x} \vec{v}(x) \\
& +\frac{x}{25 M g^{\prime} Y_{R} 10^{20} G}\left\langle\vec{E}_{Y}(x) \cdot \partial_{x} \vec{v}(x)\right\rangle, \\
& \Lambda_{L}(x)=-\frac{6 g^{\prime} Y_{L}}{8 \pi^{2}} \frac{x}{5000} \frac{\eta_{e_{L}}(x)}{10^{2} G}\left[\left\langle\vec{v}(x) . \partial_{x} \vec{B}_{Y}(x)\right\rangle\right. \\
& \left.+\left\langle\frac{\vec{v}(x) \cdot \vec{B}_{Y}(x)}{x}\right\rangle+\left\langle\vec{B}_{Y}(x) \cdot \partial_{x} \vec{v}(x)\right\rangle\right] \\
& -\left[\frac{36 M}{4 \pi^{2}} \eta_{e_{L}}^{2}(x)+\frac{1}{12 M}\right] k \vec{v}(x) \cdot \partial_{x} \vec{v}(x) \\
& +\frac{x}{25 M g^{\prime} Y_{L} 10^{20} G}\left\langle\vec{E}_{Y}(x) . \partial_{x} \vec{v}(x)\right\rangle,
\end{aligned}
$$

where $M=2 \pi^{2} g^{*} / 45$, and the coefficients $C_{i}, i=1, \ldots, 8$, are

$$
\begin{aligned}
& C_{1}=0.00096\left(\frac{k}{10^{-7}}\right) \alpha_{Y}, \\
& C_{2}=865688 \alpha_{Y}^{2}, \\
& C_{3}=0.71488\left(\frac{k}{10^{-7}}\right) \alpha_{Y}^{3 / 2}, \\
& C_{4}=17152.7\left(\frac{k}{10^{-7}}\right) \alpha_{Y}^{3 / 2},
\end{aligned}
$$




$$
\begin{aligned}
& C_{5}=0.356\left(\frac{k}{10^{-7}}\right)^{2}, \\
& C_{6}=3.18373 \times 10^{8} \alpha_{Y}\left(\frac{k}{10^{-7}}\right), \\
& C_{7}=262.9 \times 10^{20} \sqrt{\alpha_{Y}}\left(\frac{k}{10^{-7}}\right)^{2}, \\
& C_{8}=63 \times 10^{25} \sqrt{\alpha_{Y}}\left(\frac{k}{10^{-7}}\right)^{2},
\end{aligned}
$$

and $\alpha_{Y}=g^{\prime 2} / 4 \pi \simeq 0.01$ is the fine-structure constant for the $\mathrm{U}_{\mathrm{Y}}(1)$. We now choose the profile of temperature fluctuation $\beta[x(T)]=\Delta T^{2} / T^{2}$, as defined in Eq. (2.13) and the paragraph below it, to be a Gaussian function of $x$ :

$\beta(x)=\frac{\beta_{0}}{b \sqrt{2 \pi}} \exp \left[-\frac{\left(x-x_{0}\right)^{2}}{2 b^{2}}\right]$,

where $\beta_{0}$ is the amplitude multiplying the normalized Gaussian distribution, and $x=\left(t / t_{E W}\right)=\left(T_{E W} / T\right)^{2}$, as defined before. The profile of the vorticity fluctuation must have an overlap with that of temperature fluctuation, in order to produce any effect. For simplicity, we choose the two profiles to be identical. That is,

$\omega(x)=k^{\prime} v(x)=\frac{k^{\prime} v_{0}}{b \sqrt{2 \pi}} \exp \left[-\frac{\left(x-x_{0}\right)^{2}}{2 b^{2}}\right]$,

where $v_{0}$ is the amplitude of the velocity fluctuation. We should mention that the occurrence of any fluctuation in a plasma in a quasi-equilibrium state would normally trigger a restoring response originating from dissipative effects, such as viscous effects. Here, for simplicity we assume that the combined results of the original fluctuations and the ensuing dissipative effects have the profiles given by Eqs. (2.30) and (2.31).

The majority of analysis presented in the following section is for a single pulse for temperature and vorticity, as stated above, which, as we shall show, produce matter-antimatter asymmetries and helical hypermagnetic field. However, at the end of the next section we present the results for two sets of successive pulses, the latter one having the same temperature profile but with negative amplitude, showing that the first pulse is the main determinant of the outcome.

\section{Numerical solution}

In this section, we obtain the numerical solutions of the evolution equations. As mentioned earlier, we investigate the effects of the temperature fluctuations of right-handed electrons, in the presence of vorticity, on the generation and evolution of the hypermagnetic field and the matter-antimatter asymmetries, in the temperature range $100 \mathrm{GeV} \leq T \leq$
$10 \mathrm{TeV}$. We consider the temperature fluctuations as small Gaussian distributions in $x$, as shown in Eq. (2.30), that occur close to the EWPT. As for the vorticity field, we consider small fluctuations, whose profiles, as shown in Eq. (2.31), coincide with those of the temperature fluctuations. We also investigate the cases with constant vorticity fields for comparison. As we shall show, the former is much more interesting and will be the focus of our work, since it is not only physically more realistic, but also could yield orders of magnitude larger results for the asymmetries and the hypermagnetic field. In the following, we solve the evolution equations by considering the comoving wave number as $k=10^{-7}$, and setting the initial values of the hypermagnetic field amplitude and the matter-antimatter asymmetries to zero, i.e. $B_{Y}^{(0)}=0$, and $\eta_{e_{R}}^{(0)}=\eta_{e_{L}}^{(0)}=\eta_{B}^{(0)}=0$.

For our first case, we solve the coupled differential equations with the initial conditions, $v_{0}=10^{-5}, b=2 \times 10^{-4}$, and $x_{0}=45 \times 10^{-5}$, for various values of the amplitude of temperature fluctuations $\beta_{0}$, and present the results in Fig. 1. As can be seen in the figure, the simultaneous occurrence of small vorticity fluctuation and temperature fluctuation for the right handed electrons leads to the generation of strong hypermagnetic fields which then produce the matterantimatter asymmetries, all starting from zero initial values. It can be seen that by increasing the amplitude of the temperature fluctuation, the maximum and the final values of the hypermagnetic field amplitude, as well as the matterantimatter asymmetries, increase. We have found that in our model signs of the matter-antimatter asymmetries produced and the helicity of hypermagnetic and vorticity fields, are always opposite. This is a manifestation of the generalized charge conservation as stated in Eq. (4.25) of Appendix B. ${ }^{5}$ The Chern-Simons configuration that we have chosen for the hypermagnetic and vorticity fields has negative helicity. Figure 1 also shows that the hypermagnetic field amplitude grows to its maximum value of about $10^{21} \mathrm{G}$, then decreases due to the expansion of the Universe. We have also investigated the effects of changing the amplitude of the vorticity fluctuation, and have found similar results.

For our second case we solve the set of evolution equations with the initial conditions, $v_{0}=10^{-5}, \beta_{0}=5 \times 10^{-4}$, and $x_{0}=45 \times 10^{-5}$, for various values of the width or duration of both fluctuations $b$, and show the results in Fig. 2. As can be seen, by decreasing the width of the Gaussian function, the maximum and the final values of the hypermagnetic field amplitude, and the baryon asymmetry increase. ${ }^{6}$

\footnotetext{
5 In fact, the second and fourth terms in Eq. (4.25) are negligible in this study.

${ }^{6}$ We have also used a few other profiles, the results of which we can summarize as follows. For smooth profiles with the same normalization, the results are mainly dependent on the widths and not on the precise
} 


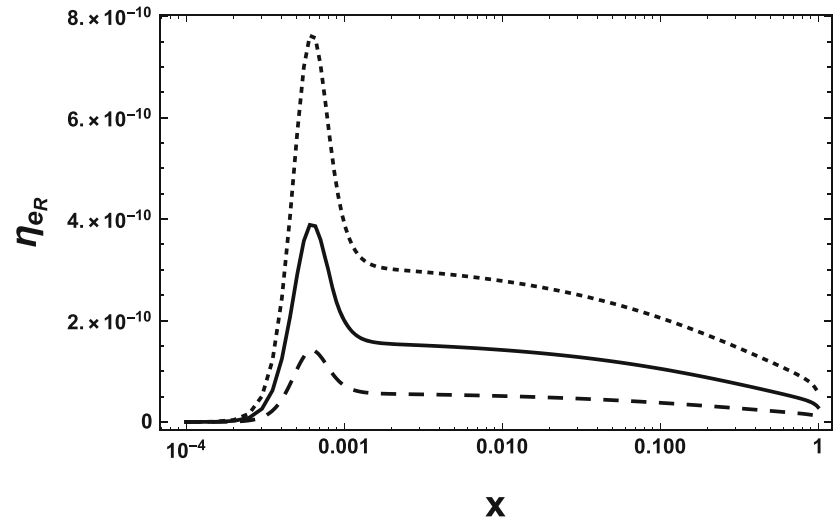

(a)

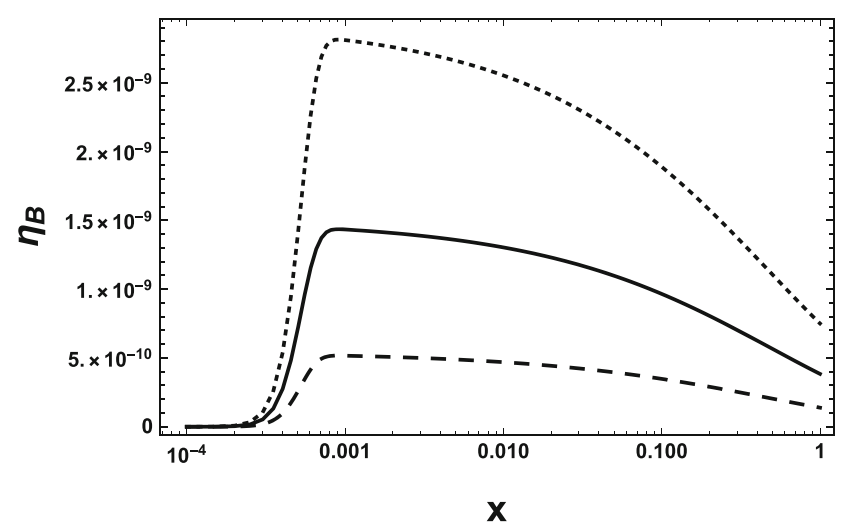

(c)

Fig. 1 Time plots of: a the right-handed electron asymmetry $\eta_{e_{R}}$, b the left-handed electron asymmetry $\eta_{e_{L}}$, $\mathbf{c}$ the baryon asymmetry $\eta_{B}$, and $\mathbf{d}$ the hypermagnetic field amplitude $B_{Y}$, for various values of the amplitude of temperature fluctuation of $e_{R}$. The initial conditions are:

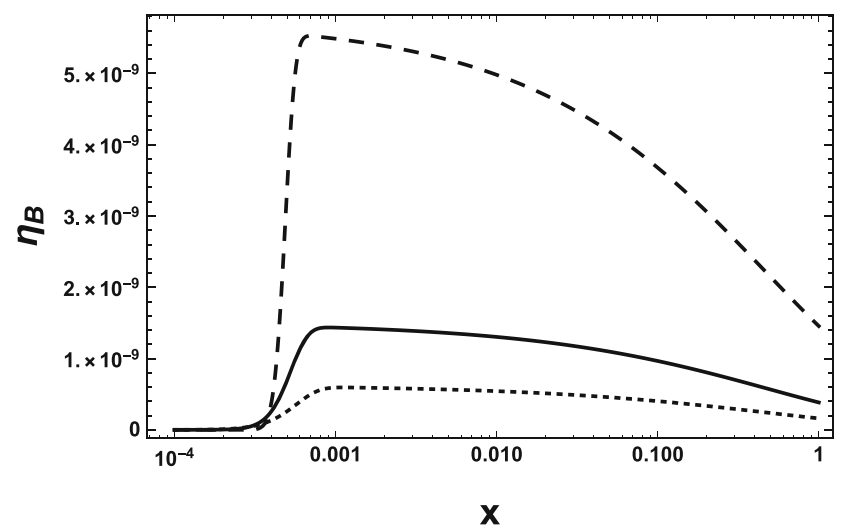

(a)

Fig. 2 Time plots of: a the baryon asymmetry $\eta_{B}$, and $\mathbf{b}$ the hypermagnetic field amplitude $B_{Y}$, for various values of the width of fluctuations. The initial conditions are: $k=10^{-7}, B_{Y}^{(0)}=0, \eta_{e_{R}}^{(0)}=\eta_{e_{L}}^{(0)}=\eta_{B}^{(0)}=0$,

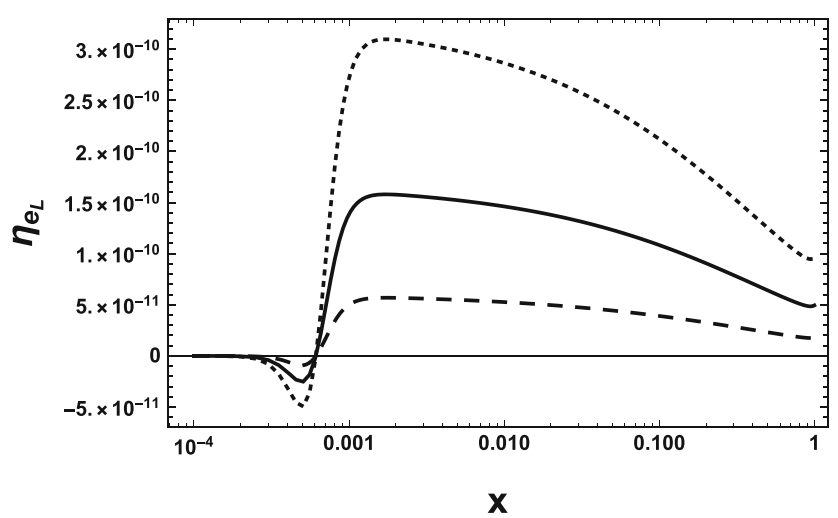

(b)

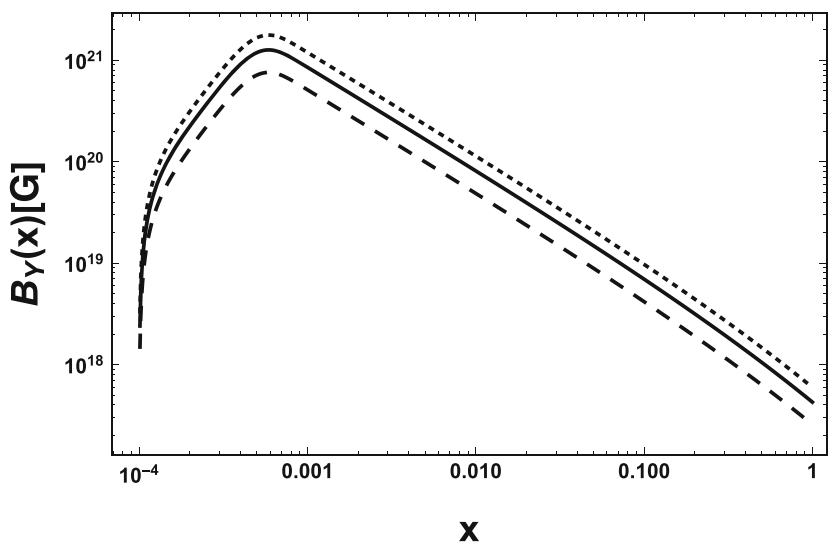

(d)

$k=10^{-7}, B_{Y}^{(0)}=0, \eta_{e_{R}}^{(0)}=\eta_{e_{L}}^{(0)}=\eta_{B}^{(0)}=0, v_{0}=10^{-5}, b=2 \times 10^{-4}$, and $x_{0}=45 \times 10^{-5}$. The dashed line is for $\beta_{0}=3 \times 10^{-4}$, the solid line is for $\beta_{0}=5 \times 10^{-4}$, and the dotted line is for $\beta_{0}=7 \times 10^{-4}$

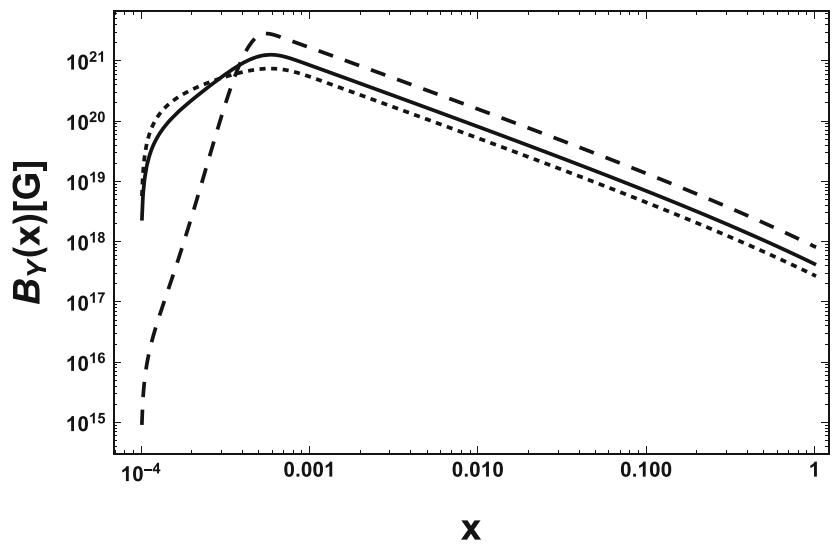

(b)

$v_{0}=10^{-5}, \beta_{0}=5 \times 10^{-4}$, and $x_{0}=45 \times 10^{-5}$. The dotted line is obtained for $b=3 \times 10^{-4}$, the solid line for $b=2 \times 10^{-4}$, the dashed line for $b=10^{-4}$ 


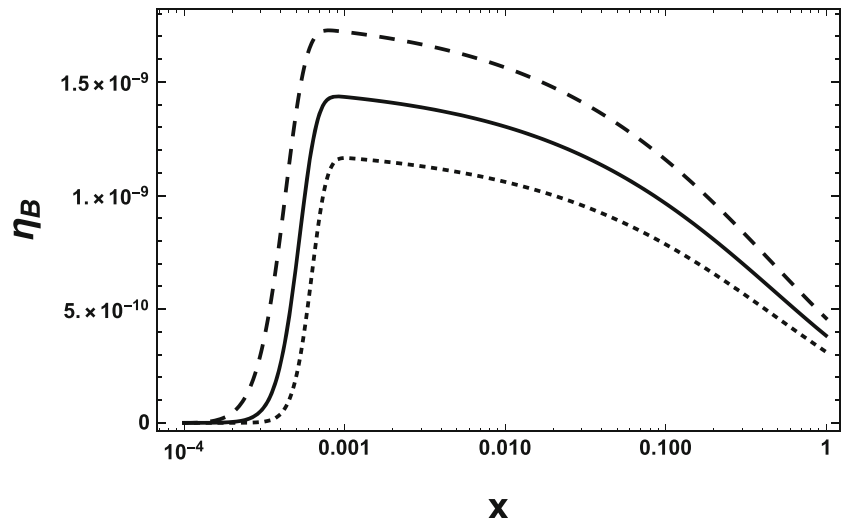

(a)

Fig. 3 Time plots of: a the baryon asymmetry $\eta_{B}$, and $\mathbf{b}$ the hypermagnetic field amplitude $B_{Y}$, for various values of the time of fluctuations. The initial conditions are: $k=10^{-7}, B_{Y}^{(0)}=0, \eta_{e_{R}}^{(0)}=\eta_{e_{L}}^{(0)}=\eta_{B}^{(0)}=0$,

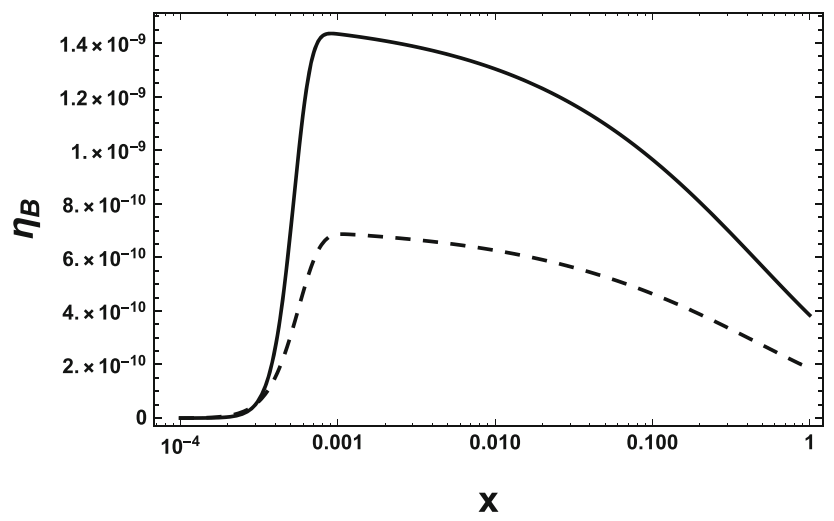

(a)

Fig. 4 Time plots of: a the baryon asymmetry $\eta_{B}$, and $\mathbf{b}$ the hypermagnetic field amplitude $B_{Y}$, for two different vorticity configurations. The initial conditions are: $k=10^{-7}, B_{Y}^{(0)}=0, \eta_{e_{R}}^{(0)}=\eta_{e_{L}}^{(0)}=\eta_{B}^{(0)}=0$,

For our third case, we solve the coupled equations with the initial conditions, $v_{0}=10^{-5}, b=2 \times 10^{-4}$, and $\beta_{0}=$ $5 \times 10^{-4}$, for various values of center time of the fluctuations $x_{0}$, and present the results in Fig. 3. As can be seen, when the fluctuations occur at an earlier time or higher temperature, the maxima and the final amplitudes of the hypermagnetic fields increase, and as a result, the matter-antimatter asymmetries increase as well.

For our fourth and final case, we solve the set of evolution equations, with the initial conditions $\beta_{0}=5 \times 10^{-4}, b=$ $2 \times 10^{-4}$ and $x_{0}=45 \times 10^{-5}$, for two different vorticity configurations. First configuration is a vorticity fluctuation

functional form of the profiles. However, the results usually increase by an order of magnitude for profiles functions which have discontinuities.

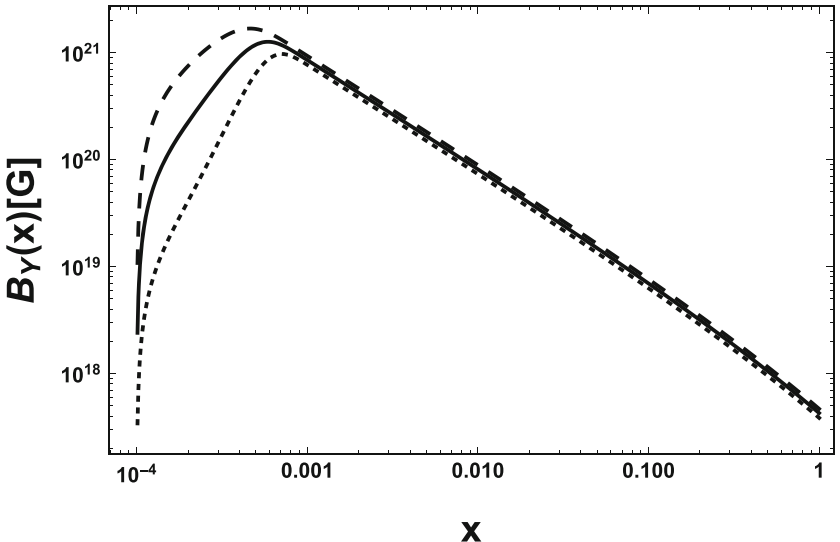

(b)

$v_{0}=10^{-5}, \beta_{0}=5 \times 10^{-4}, b=2 \times 10^{-4}$. The dotted line is obtained for $x_{0}=55 \times 10^{-5}$, the solid line for $x_{0}=45 \times 10^{-5}$, and the dashed line for $x_{0}=35 \times 10^{-5}$

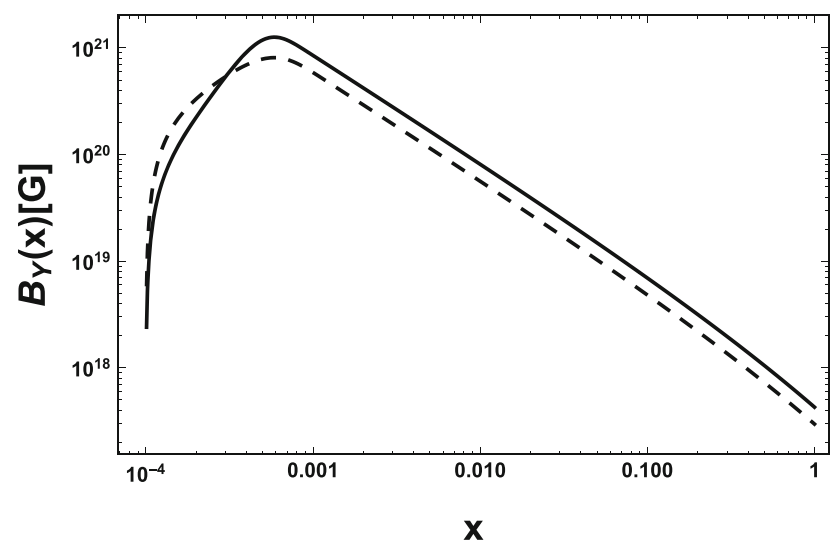

(b)

$\beta_{0}=5 \times 10^{-4}, b=2 \times 10^{-4}$, and $x_{0}=45 \times 10^{-5}$. The solid line is for vorticity fluctuation with $v_{0}=10^{-5}$, and the dashed line is for constant vorticity with $v_{0}=10^{-2}$

with amplitude $v_{0}=10^{-5}$ and $x_{0}=45 \times 10^{-5}$. Second configuration is a constant vorticity with amplitude $v_{0}=$ $10^{-2}$. The results are presented in Fig. 4. As can be seen from the figure, the general trends of the evolution curves are similar. The prominent feature of this comparison is the surprising result that a fluctuation with amplitude smaller by three orders of magnitude produces results comparable with the constant vorticity configuration.

Now we can address our assertion that $\mu / T \ll 1$ within our model, i.e., our initial conditions and results. First, note that upon using the relations $\mu_{f}=\left(6 s / T^{2}\right) \eta_{f}$ and $s=$ $\left(2 \pi^{2} g^{\star} / 45\right) T^{3}$, we obtain $\left(\mu_{f} / T\right)=\left(12 \pi^{2} g^{\star} / 45\right) \eta_{f}=$ $280.95 \eta_{f}$. The largest asymmetry in our results is obtained for $\eta_{B}$ and is shown in the Fig. 2a. This figure shows 


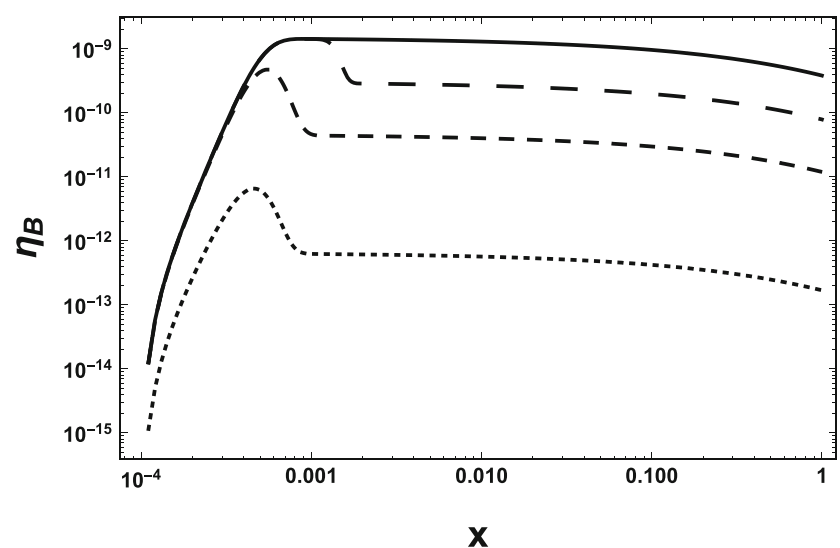

(a)

Fig. 5 Time plots of: a the baryon asymmetry $\eta_{B}$, b the hypermagnetic field amplitude $B_{Y}$ for two sets of successive and opposing fluctuations. The initial conditions are: $k=10^{-7}, B_{Y}^{(0)}=0, \eta_{e_{R}}^{(0)}=\eta_{e_{L}}^{(0)}=\eta_{B}^{(0)}=0$, $v_{0,+}=v_{0,-}=10^{-5}, b=2 \times 10^{-4}, \beta_{0,+}=-\beta_{0,-}=5 \times 10^{-4}$, and

that $\left(\eta_{B}\right)_{\max } \sim 5 \times 10^{-9}$, leading to $(\mu / T)_{\max } \sim 10^{-6}$. The condition $\mu / T \ll 1$ has been used, for example, to justify neglecting terms of $O(\mu / T)$ in Eqs. (4.5)-(4.8) in Appendix $\mathrm{A}$, leading to the expressions for $c_{\mathrm{V}}$ and $c_{\mathrm{B}}$ shown in Eqs. (2.7) and (2.8), respectively. Moreover, as shown in Appendix B, this condition, along with the assumption of non-relativistic velocity of the plasma, imply that the CME and CVE contributions to the temporal components of the four-currents in the AMHD equations are negligible within our model.

We finally address the issue of two successive pulses with opposite temperature profiles to see by how much can the second pulse negate the results of the first. For this purpose it suffices to assume that the profile of the vorticities are unchanged. To be specific, we assume

$\beta(x)=\beta_{+}(x)+\beta_{-}(x)$,

$v(x)=v_{+}(x)+v_{-}(x)$,

where

$\beta_{ \pm}(x)=\frac{ \pm \beta_{0}}{b \sqrt{2 \pi}} \exp \left[-\frac{\left(x-x_{0, \pm}\right)^{2}}{2 b^{2}}\right]$,

and

$v_{ \pm}(x)=\frac{v_{0}}{b \sqrt{2 \pi}} \exp \left[-\frac{\left(x-x_{0, \pm}\right)^{2}}{2 b^{2}}\right]$.

We consider three cases in which the time separation of the pulses $\Delta x_{0}=x_{0,+}-x_{0,-}$ are $5 b, b$ and $0.1 b$, where $b$ denotes the width of the pulses. The results are shown in Fig. 5, where we also show our results for a single pulse for comparison. As can be seen, the final values of the asymmetries generated are reduced, as compared to the single pulse case, by a factor of about 5, 50 and 1000, respectively. The final values of the

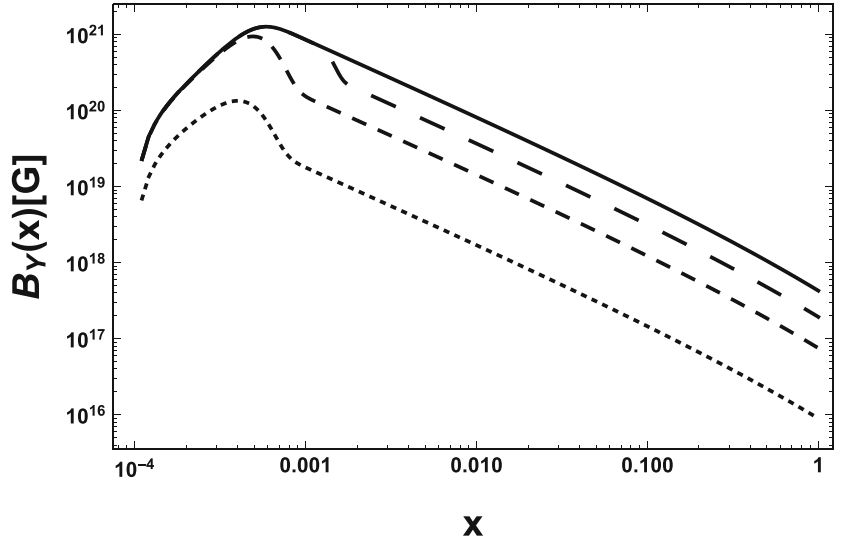

(b)

$x_{0,+}=4.5 \times 10^{-4}$. The large dashed line is for $x_{0,-}=1.45 \times 10^{-3}=$ $5 b+x_{0,+}$, the medium dashed line is for $x_{0,-}=6.5 \times 10^{-4}=b+x_{0,+}$, the dotted line is for $x_{0,-}=4.7 \times 10^{-4}=0.1 b+x_{0,+}$, and the solid line is obtained in the absence of the second set of fluctuations

hypermagnetic field generated are reduced by square root of values stated above. It is interesting to note that even in the case $\Delta x_{0}=0.1 b$, the model produced $\eta_{B} \simeq 10^{-13}$, a value which can be increased easily by increasing $\beta_{0}, v_{0}$, and/or decreasing $b$.

\section{Conclusion}

In this study, we have investigated the contribution of the temperature-dependent CVE to the generation and evolution of the hypermagnetic fields and the matter-antimatter asymmetries, in the symmetric phase of the early Universe and in the temperature range $100 \mathrm{GeV} \leq T \leq 10 \mathrm{TeV}$. The $\mathrm{CVE}$ has two possible sources in a vortical plasma, one from the chiralities and the other from the temperature of the particles. The former has been investigated in the literature much more than the latter. Here, we have focused on the latter in the form of transient temperature fluctuations, and have shown its important role in the production and evolution of the hypermagnetic fields and the matter-antimatter asymmetries. The transient fluctuations that we have considered are in the form of sharp Gaussian shaped pulses. In particular, we have shown that small simultaneous and transient fluctuations of vorticity about zero background value and temperature of some matter degrees of freedom about the equilibrium temperature of the plasma, close to the EWPT, can generate strong hypermagnetic fields and large matter-antimatter asymmetries, even in the absence of any initial seed for the hypermagnetic field or any initial matter-antimatter asymmetries. Furthermore, we have shown that, an increase in the amplitude of temperature or vorticity fluctuations leads to the 
production of stronger hypermagnetic fields, and therefore, larger matter-antimatter asymmetries. This outcome has not been observed in any of the previous studies. In some studies which only take the CME into account, either an initially strong hypermagnetic field produces matter-antimatter asymmetries, or initial large matter-antimatter asymmetries strengthen a preexisting seed of hypermagnetic field [1719]. In some other studies which also include the CVE and assume large initial chiralities in the vortical plasma, a seed of the hypermagnetic field is produced which then grows due to the CME [20].

In this work, we have considered a simple monochromatic helical configuration for the vorticity and hypermagnetic fields with a negative helicity, which ensures the production of the desired positive matter-antimatter asymmetries. Furthermore, we have shown that, either an increase in the amplitude of the temperature or vorticity fluctuations, or a decrease in their widths leads to the production of stronger hypermagnetic fields, and therefore, larger matter-antimatter asymmetries. We have also shown that fluctuations in vorticity are several orders of magnitude more productive than constant vorticity. Within our model, the temperature-dependent CVE is the dominant effect as compared to the CME. We have also shown that when there are two sets of successive pulses with opposite temperature profiles, the first pulse is the main determinant of the outcome. A generalization of this work would be a stochastic analysis of fluctuations of both temperature and vorticity.

Data Availability Statement This manuscript has no associated data or the data will not be deposited. [Authors' comment: Our generated data is in the form of the output of a Fortran program, but is not deposited.]

Open Access This article is licensed under a Creative Commons Attribution 4.0 International License, which permits use, sharing, adaptation, distribution and reproduction in any medium or format, as long as you give appropriate credit to the original author(s) and the source, provide a link to the Creative Commons licence, and indicate if changes were made. The images or other third party material in this article are included in the article's Creative Commons licence, unless indicated otherwise in a credit line to the material. If material is not included in the article's Creative Commons licence and your intended use is not permitted by statutory regulation or exceeds the permitted use, you will need to obtain permission directly from the copyright holder. To view a copy of this licence, visit http://creativecomm ons.org/licenses/by/4.0/.

Funded by SCOAP ${ }^{3}$.

\section{Appendix A}

In this appendix we present the expressions for the chiral vorticity and helicity coefficients, $c_{\mathrm{V}}$ and $c_{\mathrm{B}}$ given by Eqs. (2.7), and (2.8). First, we start with the relevant and well known expressions in the broken phase. In relativistic hydrodynamics, the energy and number currents can flow separately in the presence of dissipative processes, therefore the definition of the flow will not be trivial [69]. Some common hydrodynamic frames in which the equations of AMHD may be formulated include the Landau-Lifshitz (or energy) frame [70], the Eckart (or conserved charge/particle) frame [71], and the more recently introduced anomalous "no-drag" frame [72]. In the latter, as its name suggests, a stationary obstacle experiences no drag, even when the energy and charge currents are present. In the Landau-Lifshitz frame, the energymomentum tensor $T^{\mu \nu}$ and the total electric current $J^{\mu}$ for a plasma consisting of a single species of massless fermions of both chiralities are given by

$$
\begin{aligned}
T^{\mu \nu} & =(\rho+p) u^{\mu} u^{\nu}-p g^{\mu \nu}+\frac{1}{4} g^{\mu \nu} F^{\alpha \beta} F_{\alpha \beta}-F^{v \sigma} F_{\sigma}^{\mu}+\tau^{\mu \nu} \\
J^{\mu} & =\rho_{\mathrm{el}} u^{\mu}+J_{\mathrm{cm}}^{\mu}+J_{\mathrm{cv}}^{\mu}+v^{\mu} \\
J_{\mathrm{cm}}^{\mu} & =\left(Q_{\mathrm{R}} \xi_{\mathrm{B}, \mathrm{R}}+Q_{\mathrm{L}} \xi_{\mathrm{B}, \mathrm{L}}\right) B^{\mu}=c_{\mathrm{B}} B^{\mu} \\
J_{\mathrm{cv}}^{\mu} & =\left(Q_{\mathrm{R}} \xi_{\mathrm{v}, \mathrm{R}}+Q_{\mathrm{L}} \xi_{\mathrm{v}, \mathrm{L}}\right) \omega^{\mu}=c_{\mathrm{v}} \omega^{\mu}
\end{aligned}
$$

where $F_{\alpha \beta}=\nabla_{\alpha} A_{\beta}-\nabla_{\beta} A_{\alpha}$ is the field strength tensor, $p$ and $\rho$ are the pressure and the energy density of the plasma, $\rho_{\mathrm{el}}$ is the electric charge density, $u^{\mu}=\gamma(1, \vec{v} / R)$ is the four-velocity of the plasma normalized such that $u^{\mu} u_{\mu}=1$, and $\gamma$ is the Lorentz factor. ${ }^{7}$ In the above equations, $v^{\mu}$ and $\tau^{\mu \nu}$ denote the electric diffusion current and viscous stress tensor, respectively [3], $Q_{R}\left(Q_{L}\right)$ denotes the electric charges of the right-handed (left-handed) fermions, $B^{\mu}=\left(\epsilon^{\mu \nu \rho \sigma} / 2 R^{3}\right) u_{\nu} F_{\rho \sigma}$ is the magnetic field four-vector, and $\omega^{\mu}=\left(\epsilon^{\mu \nu \rho \sigma} / R^{3}\right) u_{v} \nabla_{\rho} u_{\sigma}$ is the vorticity four-vector, with the totally anti-symmetric Levi-Civita tensor density specified by $\epsilon^{0123}=-\epsilon_{0123}=1$.

Furthermore, in the Landau-Lifshitz frame, the CME and CVE coefficients for chiral fermions are given as [3,49-53]

$$
\begin{aligned}
\xi_{\mathrm{B}, \mathrm{R}} & =\frac{Q_{\mathrm{R}} \mu_{\mathrm{R}}}{4 \pi^{2}}\left[1-\frac{1}{2} \frac{n_{\mathrm{R}} \mu_{\mathrm{R}}}{\rho+p}\right]-\frac{1}{24} \frac{n_{\mathrm{R}} T^{2}}{\rho+p}, \\
\xi_{\mathrm{B}, \mathrm{L}} & =-\frac{Q_{\mathrm{L}} \mu_{\mathrm{L}}}{4 \pi^{2}}\left[1-\frac{1}{2} \frac{n_{\mathrm{L}} \mu_{\mathrm{L}}}{\rho+p}\right]+\frac{1}{24} \frac{n_{\mathrm{L}} T^{2}}{\rho+p}, \\
\xi_{\mathrm{v}, \mathrm{R}} & =\frac{\mu_{\mathrm{R}}^{2}}{8 \pi^{2}}\left[1-\frac{2}{3} \frac{n_{\mathrm{R}} \mu_{\mathrm{R}}}{\rho+p}\right]+\frac{1}{24} T^{2}\left[1-\frac{2 n_{\mathrm{R}} \mu_{\mathrm{R}}}{\rho+p}\right], \\
\xi_{\mathrm{v}, \mathrm{L}} & =-\frac{\mu_{\mathrm{L}}^{2}}{8 \pi^{2}}\left[1-\frac{2}{3} \frac{n_{\mathrm{L}} \mu_{\mathrm{L}}}{\rho+p}\right]-\frac{1}{24} T^{2}\left[1-\frac{2 n_{\mathrm{L}} \mu_{\mathrm{L}}}{\rho+p}\right] .
\end{aligned}
$$

where $T$ is the temperature and $n_{\mathrm{R}}\left(n_{\mathrm{L}}\right)$ is the right-handed (left-handed) charge density. ${ }^{8}$ As we shall show explicitly in Sect. $3, \mu_{\mathrm{R}, \mathrm{L}} / T \ll 1$ within our model, i.e., our initial

\footnotetext{
7 Note that the self-consistency of our calculation in which the diagonal Einstein tensor obtained from the FRW metric is used implies that not only should the electromagnetic field density be small compared to the energy density of the Universe [73], but also the bulk velocity should obey the condition $|\vec{v}| \ll 1$, or equivalently $\gamma \simeq 1$ and $u^{\mu} \simeq(1, \vec{v} / R)$. 8 The charge density $n$ is the difference between the particle and antiparticle charge densities.
} 
conditions and results. Hence, $n_{\mathrm{R}, \mathrm{L}} \simeq \frac{1}{6} \mu_{\mathrm{R}, \mathrm{L}} T^{2}$ and $\rho=$ $3 p \simeq \frac{\pi^{2}}{30} g^{*} T^{4}$, and Eqs. (4.5)-(4.8) may be simplified as follows

$$
\begin{aligned}
\xi_{\mathrm{B}, \mathrm{R}} & \simeq \frac{Q_{\mathrm{R}} \mu_{\mathrm{R}}}{4 \pi^{2}}, \\
\xi_{\mathrm{B}, \mathrm{L}} & \simeq-\frac{Q_{\mathrm{L}} \mu_{\mathrm{L}}}{4 \pi^{2}}, \\
\xi_{\mathrm{v}, \mathrm{R}} & \simeq \frac{\mu_{\mathrm{R}}^{2}}{8 \pi^{2}}+\frac{1}{24} T^{2}, \\
\xi_{\mathrm{v}, \mathrm{L}} & \simeq-\frac{\mu_{\mathrm{L}}^{2}}{8 \pi^{2}}-\frac{1}{24} T^{2} .
\end{aligned}
$$

Moreover, since $\mu / T \ll 1$, we will consider only the Ohmic part of the diffusion current $v^{\mu}=\sigma\left[E^{\mu}+T\left(u^{\mu} u^{\nu}-\right.\right.$ $\left.\left.g^{\mu \nu}\right) \nabla_{\nu}\left(\frac{\mu}{T}\right)\right]$ given by the first term, where $\sigma=\sigma_{R}+\sigma_{L}$ is the electrical conductivity, and $E^{\mu}=F^{\mu v} u_{v}$ is the electric field four-vector. In order to carry over these results to the symmetric phase of the early Universe, it suffices to replace the electromagnetic field by the hypercharge gauge field and the electric charges of different particle species by their relevant hypercharges. Taking into account all three generations of leptons and quarks, we can easily obtain the chiral vorticity and helicity coefficients $c_{\mathrm{V}}$ and $c_{\mathrm{B}}$, given by Eqs. (2.7), and (2.8), using Eqs. (4.9)-(4.12). The four-vectors $B^{\mu}, \omega^{\mu}$, $a^{\mu}$, and $E^{\mu}$ in the limit $v \ll 1$ are given below,

$$
\begin{aligned}
& B^{\mu}=\gamma\left(\vec{v} \cdot \vec{B}, \frac{\vec{B}-\vec{v} \times \vec{E}}{R}\right) \simeq\left(\vec{v} \cdot \vec{B}, \frac{\vec{B}}{R}\right) \\
& \omega^{\mu}=\gamma\left(\vec{v} \cdot \vec{\omega}, \frac{\vec{\omega}-\vec{v} \times \vec{a}}{R}\right) \simeq\left(\vec{v} \cdot \vec{\omega}, \frac{\vec{\omega}}{R}\right) \\
& a^{\mu}=\gamma\left(\vec{v} \cdot \vec{a}, \frac{\vec{a}+\vec{v} \times \vec{\omega}}{R}\right) \simeq\left(\vec{v} \cdot \vec{a}, \frac{\vec{a}}{R}\right) \\
& E^{\mu}=\gamma\left(\vec{v} \cdot \vec{E}, \frac{\vec{E}+\vec{v} \times \vec{B}}{R}\right) \simeq\left(\vec{v} \cdot \vec{E}, \frac{\vec{E}+\vec{v} \times \vec{B}}{R}\right)
\end{aligned}
$$

where $a^{\mu}=\Omega^{\mu v} u_{v}$ is acceleration four-vector, $\Omega_{\mu v}=$ $\nabla_{\mu} u_{\nu}-\nabla_{\nu} u_{\mu}$ is vorticity tensor, $a^{i}=R \Omega^{0 i}$ is three vector acceleration. We have also used the assumption $\partial_{t} \sim \vec{\nabla} \cdot \vec{v}$ in the derivative expansion of the hydrodynamics, so $\vec{v} \times \vec{E} \simeq$ $v^{2} \vec{B}$ and we have ignored the terms of $O\left(v^{2}\right)$.

\section{Appendix B}

In this appendix we start with the basic expression for the anomaly given by Eq. (2.20) and obtain its explicit form which eventually leads to Eqs. (2.21), (2.24) and (2.25). The equations of AMHD consist of energy-momentum conservation, Maxwell's equations and the anomaly relations. These equations may be expressed covariantly as

$$
\nabla_{\mu} T^{\mu \nu}=0,
$$

$$
\begin{aligned}
\nabla_{\mu} F^{\mu v} & =J^{v} \\
\nabla_{\mu} \tilde{F}^{\mu v} & =0 \\
\nabla_{\mu} j_{\mathrm{R}, \mathrm{L}}^{\mu} & =C_{\mathrm{R}, \mathrm{L}} E_{\mu} B^{\mu},
\end{aligned}
$$

where $\tilde{F}^{\mu \nu}=\frac{1}{2 R^{3}} \epsilon^{\mu \nu \rho \sigma} F_{\rho \sigma}$ is the dual field strength tensor, $C_{R, L}$ are the corresponding right- and left- handed anomaly coefficients, and $j_{R, L}^{\mu}$ are the fermionic currents given as follows

$$
\begin{aligned}
& j_{\mathrm{R}}^{\mu}=n_{\mathrm{R}} u^{\mu}+\xi_{\mathrm{B}, \mathrm{R}} B^{\mu}+\xi_{\mathrm{v}, \mathrm{R}} \omega^{\mu}+V_{R}^{\mu}, \\
& j_{\mathrm{L}}^{\mu}=n_{\mathrm{L}} u^{\mu}+\xi_{\mathrm{B}, \mathrm{L}} B^{\mu}+\xi_{\mathrm{v}, \mathrm{L}} \omega^{\mu}+V_{L}^{\mu},
\end{aligned}
$$

where $n_{\mathrm{R}, \mathrm{L}}$ and $V_{\mathrm{R}, \mathrm{L}}^{\mu}$ are the chiral charge density and chiral particle diffusion current, respectively [3,51-53]. The anomaly equations given above can be written out as

$\partial_{t} j_{(\mathrm{R}, \mathrm{L})}^{0}+\frac{1}{R} \vec{\nabla} \cdot \vec{j}_{(R, L)}+3 H j_{(\mathrm{R}, \mathrm{L})}^{0}=C_{(\mathrm{R}, \mathrm{L})} E_{\mu} B^{\mu}$,

where

$j_{(R, L)}^{0}=n_{(\mathrm{R}, \mathrm{L})}+\xi_{\mathrm{B},(\mathrm{R}, \mathrm{L})} \vec{v} \cdot \vec{B}+\xi_{\mathrm{v},(\mathrm{R}, \mathrm{L})} \vec{v} \cdot \vec{\omega}+V_{R, L}^{0}($

Upon taking the spatial average of Eq. (4.21), the boundary term vanishes and we obtain ${ }^{9}$

$$
\begin{aligned}
\partial_{t} n_{(\mathrm{R}, \mathrm{L})}+3 H n_{(\mathrm{R}, \mathrm{L})}= & -\left[\partial_{t}+3 H\right]\left[\xi_{\mathrm{B},(\mathrm{R}, \mathrm{L})}\langle\vec{v} \cdot \vec{B}\rangle+\xi_{\mathrm{V},(\mathrm{R}, \mathrm{L})}\langle\vec{v} \cdot \vec{\omega}\rangle\right. \\
& \left.+\frac{\sigma_{\mathrm{R}, \mathrm{L}}}{Q_{\mathrm{R}, \mathrm{L}}}\langle\vec{v} \cdot \vec{E}\rangle\right]-C_{R, L}\langle\vec{E} \cdot \vec{B}\rangle .
\end{aligned}
$$

Using the relation $\dot{s} / s=-3 H$, the anomaly equation reduces to the simplified form

$$
\begin{aligned}
\partial_{t}\left(\frac{n_{(\mathrm{R}, \mathrm{L})}}{s}\right)= & -\partial_{t}\left[\frac{\xi_{\mathrm{B},(\mathrm{R}, \mathrm{L})}}{s}\langle\vec{v} \cdot \vec{B}\rangle+\frac{\xi_{\mathrm{v},(\mathrm{R}, \mathrm{L})}}{s}\langle\vec{v} \cdot \vec{\omega}\rangle\right. \\
& \left.+\frac{\sigma_{\mathrm{R}, \mathrm{L}}}{Q_{\mathrm{R}, \mathrm{L}} s}\langle\vec{v} \cdot \vec{E}\rangle\right]-\frac{C_{\mathrm{R}, \mathrm{L}}}{s}\langle\vec{E} \cdot \vec{B}\rangle .
\end{aligned}
$$

It is worth mentioning that since $\left(\partial_{t}+3 H\right)\langle\vec{A} \cdot \vec{B}\rangle=-2\langle\vec{E}$. $\vec{B}\rangle$, Eq. (4.24) can be rewritten in the following form

$$
\begin{gathered}
\partial_{t}\left[\eta_{R, L}+\frac{\xi_{\mathrm{B},(\mathrm{R}, \mathrm{L})}}{s}\langle\vec{v} \cdot \vec{B}\rangle+\frac{\xi_{\mathrm{v},(\mathrm{R}, \mathrm{L})}}{s}\langle\vec{v} \cdot \vec{\omega}\rangle\right. \\
\left.+\frac{\sigma_{(R, L)}}{Q_{(R, L)} s}\langle\vec{v} \cdot \vec{E}\rangle-\frac{C_{\mathrm{R}, \mathrm{L}}}{2 s}\langle\vec{A} \cdot \vec{B}\rangle\right]=0,
\end{gathered}
$$

which clearly reveals the conservation of a generalized charge [7,51].

Using Eqs. (4.24), (4.9)-(4.12) and $x=\left(T_{\mathrm{EW}} / T\right)^{2}=$ $t / t_{\mathrm{EW}}$, we obtain

$$
\begin{aligned}
& \frac{d \eta_{\mathrm{R}}}{d x}=\frac{1}{\left[1+\frac{6 Q_{\mathrm{R}}}{4 \pi^{2}} \frac{\langle\vec{v} \cdot \vec{B}\rangle}{10^{20} G} \frac{x}{5000}-\frac{36 M}{4 \pi^{2}} \eta_{\mathrm{R}} k v^{2}\right]} \\
& \quad \times\left[-\frac{6 Q_{\mathrm{R}}}{4 \pi^{2}} \frac{x}{5000} \frac{\eta_{\mathrm{R}}}{10^{20} G}\left[\left\langle\vec{v} \cdot \partial_{x} \vec{B}\right\rangle+\left\langle\frac{\vec{v} \cdot \vec{B}}{x}\right\rangle+\left\langle\vec{B} \cdot \partial_{x} \vec{v}\right\rangle\right]\right.
\end{aligned}
$$

$\overline{{ }^{9} \text { Note that } E_{\mu}} B^{\mu}=(\vec{v} \cdot \vec{E})(\vec{v} \cdot \vec{B})-(\vec{E}+\vec{v} \times \vec{B}) \cdot(\vec{B}-\vec{v} \times \vec{E})=$ $-\vec{E} \cdot \vec{B}+O\left(v^{2}\right)$, and we have ignored the terms of $O\left(v^{2}\right)$. 


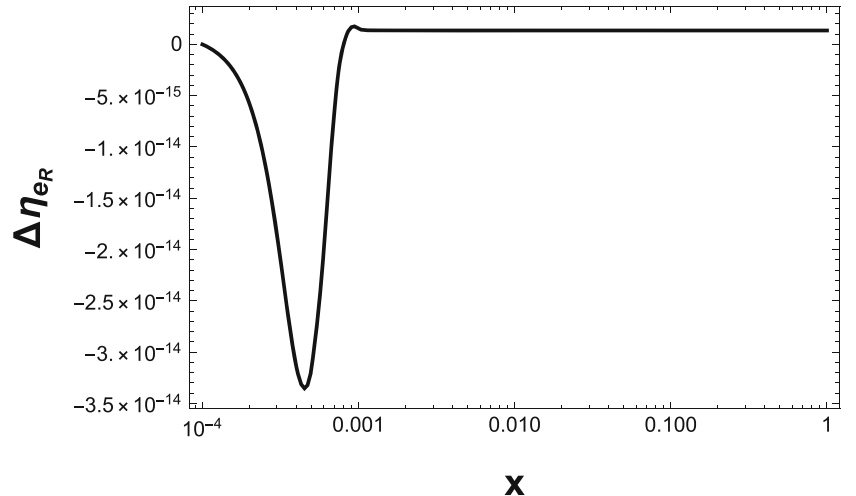

(a)

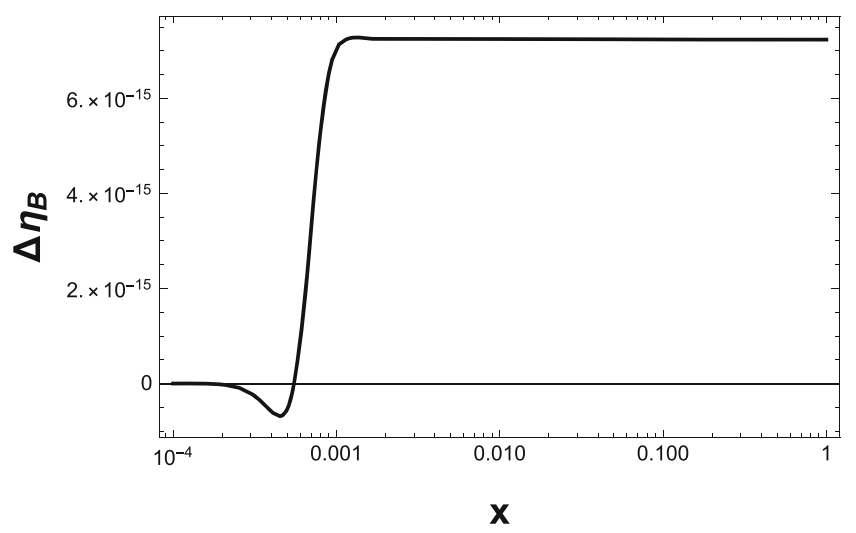

(c)

Fig. 6 Time plots of the differences between the results with and without the inclusion of the temporal components of the CVE, CME and diffusion currents given by the last three terms of Eq. (4.22): a the righthanded electron asymmetry $\Delta \eta_{e_{R}}$, b the left-handed electron asym-

$$
\begin{aligned}
& +\left[\frac{36 M}{4 \pi^{2}} \eta_{\mathrm{R}}^{2}+\frac{1}{12 M}\right] k \vec{v} \cdot \partial_{x} \vec{v} \\
& -\frac{x}{50 M Q_{\mathrm{R}} 10^{20} G}\left[\left\langle\vec{v} \cdot \partial_{x} \vec{E}\right\rangle+\left\langle\frac{\vec{v} \cdot \vec{E}}{x}\right\rangle+\left\langle\vec{E} \cdot \partial_{x} \vec{v}\right\rangle\right] \\
& \left.-\frac{t_{\mathrm{EW}} C_{\mathrm{R}}}{s}\langle\vec{E} \cdot \vec{B}\rangle\right], \\
& \frac{d \eta_{\mathrm{L}}}{d x}=\frac{1}{\left[1-\frac{6 Q_{\mathrm{L}}}{4 \pi^{2}} \frac{\langle\vec{v} \cdot \vec{B}\rangle}{10^{20} G} \frac{x}{5000}+\frac{36 M}{4 \pi^{2}} \eta_{\mathrm{L}} k v^{2}\right]} \\
& \times\left[\frac{6 Q_{\mathrm{L}}}{4 \pi^{2}} \frac{x}{5000} \frac{\eta_{\mathrm{L}}}{10^{20} G}\left[\left\langle\vec{v} \cdot \partial_{x} \vec{B}\right\rangle+\left\langle\frac{\vec{v} \cdot \vec{B}}{x}\right\rangle+\left\langle\vec{B} \cdot \partial_{x} \vec{v}\right\rangle\right]\right. \\
& -\left[\frac{36 M}{4 \pi^{2}} \eta_{\mathrm{L}}^{2}+\frac{1}{12 M}\right] k \vec{v} \cdot \partial_{x} \vec{v} \\
& -\frac{x}{50 M Q_{\mathrm{L}} 10^{20} G}\left[\left\langle\vec{v} \cdot \partial_{x} \vec{E}\right\rangle+\left\langle\frac{\vec{v} \cdot \vec{E}}{x}\right\rangle+\left\langle\vec{E} \cdot \partial_{x} \vec{v}\right\rangle\right] \\
& \left.-\frac{t_{\mathrm{EW}} C_{\mathrm{L}}}{s}\langle\vec{E} \cdot \vec{B}\rangle\right] \text {, }
\end{aligned}
$$

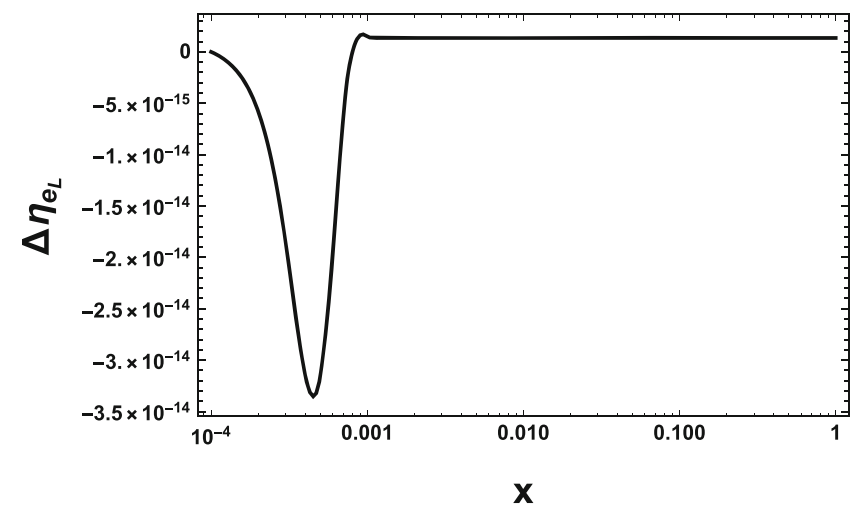

(b)

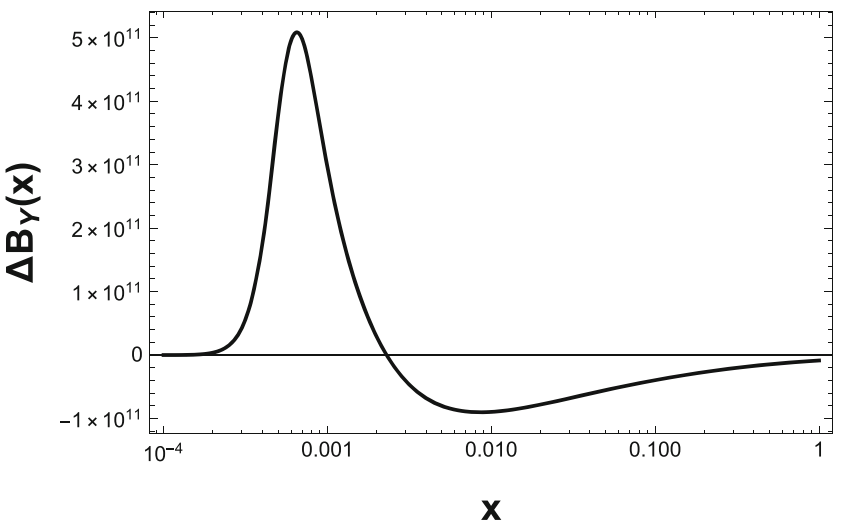

(d)

metry $\Delta \eta_{e_{L}}, \mathbf{c}$ the baryon asymmetry $\Delta \eta_{B}$, and $\mathbf{d}$ the hypermagnetic field amplitude $\Delta B_{Y}$. The initial conditions are: $k=10^{-7}, B_{Y}^{(0)}=0$, $\eta_{e_{R}}^{(0)}=\eta_{e_{L}}^{(0)}=\eta_{B}^{(0)}=0, \beta_{0}=5 \times 10^{-4}, v_{0}=10^{-5}, b=2 \times 10^{-4}$, and $x_{0}=45 \times 10^{-5}$

where $M=2 \pi^{2} g^{*} / 45$. Here the electric field $\vec{E}$ is not an independent variable and is determined by Eq. (2.18). From the approximation of a vanishing displacement current, we have $\partial_{x} \vec{E}=-\vec{E} / x$.

As is well known, in the symmetric phase of the early Universe $T>100 \mathrm{GeV}$, and in Sect. 3 we show that the condition $\mu / T \leq 10^{-6}$ holds within our model. These conditions, in conjunction with the low-velocity limit considered in this work, highly suppress the contributions of the temporal components of the CVE, CME and diffusion currents. To ascertain this claim, we have solved Eqs. (2.24)-(2.26) both with and without the additional temporal components and present the differences in Fig. 6. Comparing the scales of this figure and Fig. 1, we observe the following for the differences: $\Delta \eta / \eta \sim 10^{-4}$ and $\Delta B_{Y} / B_{Y} \sim 10^{-9}$. 


\section{References}

1. D. E. Kharzeev, Topology, magnetic field, and strongly interacting matter. Ann. Rev. Nucl. Part. Sci. 65, 193-214 (2015). arXiv:1501.01336 [hep-ph]

2. A. Vilenkin, Macroscopic parity violating effects: neutrino fluxes from rotating black holes and in rotating thermal radiation. Phys. Rev. D 20, 1807 (1979). https://doi.org/10.1103/PhysRevD.20. 1807

3. D.T. Son, P. Surowka, Hydrodynamics with triangle anomalies. Phys. Rev. Lett. 103, 191601 (2009). arXiv:0906.5044 [hep-th]

4. A.V. Sadofyev, V.I. Shevchenko, V.I. Zakharov, Notes on chiral hydrodynamics within effective theory approach. Phys. Rev. D 83, 105025 (2011). arXiv:1012.1958 [hep-th]

5. S. Pu, J. Gao, Q. Wang, A consistent description of kinetic equation with triangle anomaly. Phys. Rev. D 83, 094017 (2011). arXiv:1008.2418 [nucl-th]

6. V.P. Kirilin, A.V. Sadofyev, V.I. Zakharov, Chiral vortical effect in superfluid. Phys. Rev. D 86, 025021 (2012). arXiv:1203.6312 [hep-th]

7. A. Avdoshkin, V. P. Kirilin, A. V. Sadofyev, V. I. Zakharov, On consistency of hydrodynamic approximation for chiral media. Phys. Lett. B 755, 1-7 (2016). arXiv:1402.3587 [hep-th]

8. A. Avkhadiev, A.V. Sadofyev, Chiral vortical effect for bosons. Phys. Rev. D 96(4), 045015 (2017). arXiv: 1702.07340 [hep-th]

9. V.P. Kirilin, A.V. Sadofyev, Anomalous transport and generalized axial charge. Phys. Rev. D 96(1), 016019 (2017). arXiv:1703.02483 [hep-th]

10. O.F. Dayi, E. Kilincarslan, Quantum kinetic equation in the rotating frame and chiral kinetic theory. Phys. Rev. D 98(8), 081701 (2018). arXiv:1807.05912 [hep-th]

11. A. Vilenkin, Equilibrium parity violating current in a magnetic field. Phys. Rev. D 22, 3080 (1980). https://doi.org/10.1103/ PhysRevD.22.3080

12. D. Kharzeev, Parity violation in hot QCD: why it can happen, and how to look for it. Phys. Lett. B 633, 260 (2006). arXiv:hep-ph/0406125

13. D. Kharzeev, A. Zhitnitsky, Charge separation induced by Podd bubbles in QCD matter. Nucl. Phys. A 797, 67 (2007). arXiv:0706.1026 [hep-ph]

14. D.E. Kharzeev, L.D. McLerran, H.J. Warringa, The effects of topological charge change in heavy ion collisions: event by event $\mathrm{P}$ and CP violation. Nucl. Phys. A 803, 227 (2008). arXiv:0711.0950 [hep-ph]

15. Jian-Hua Gao, Zuo-Tang Liang, Pu Shi, Qun Wang, Xin-Nian Wang, Chiral anomaly and local polarization effect from quantum kinetic approach. Phys. Rev. Lett. 109, 32301 (2012). arXiv:1203.0725 [hep-ph]

16. D.E. Kharzeev, J. Liao, S.A. Voloshin, G. Wang, Chiral magnetic and vortical effects in high-energy nuclear collisions-a status report. Prog. Part. Nucl. Phys. 88, 1-28 (2016). arXiv:1511.04050 [hep-ph]

17. S. Rostam Zadeh, S.S. Gousheh, Contributions to the $U_{Y}(1)$ Chern-Simons term and the evolution of fermionic asymmetries and hypermagnetic fields. Phys. Rev. D 94, 056013 (2016). arXiv:1512.01942 [hep-ph]

18. S. Rostam Zadeh, S.S. Gousheh, Effects of the $U_{Y}(1)$ ChernSimons term and its baryonic contribution on matter asymmetries and hypermagnetic fields. Phys. Rev. D 95, 056001 (2017). arXiv:1607.00650 [hep-ph]

19. S. Rostam Zadeh, S.S. Gousheh, A minimal system including weak sphalerons for investigating the evolution of matter asymmetries and hypermagnetic fields. Phys. Rev. D 99, 096009 (2019). arXiv:1812.10092 [hep-ph]
20. S.Rostam Zadeh Abbaslu, S.S. Gousheh, Contribution of the chiral vortical effect to the evolution of the hypermagnetic field and the matter-antimatter asymmetry in the early Universe. Phys. Rev. D 100(11), 116022 (2019). arXiv:1908.10105 [hep-ph]

21. F. Elahi, S.R. Zadeh, Flavon magneto-baryogenesis. Phys. Rev. D 102(9). 096018 (2020). arXiv:2008.04434 [hep-ph]

22. L.M. Widrow, Origin of galactic and extragalactic magnetic fields. Rev. Mod. Phys. 74, 775 (2002). arXiv:astro-ph/0207240

23. P.P. Kronberg, Extragalactic magnetic fields. Rep. Prog. Phys. 57, 325 (1994). https://doi.org/10.1088/0034-4885/57/4/001

24. R.M. Kulsrud, R. Cen, J.P. Ostriker, D. Ryu, The protogalactic origin for cosmic magnetic fields. Astrophys. J. 480, 481 (91997). arXiv:astro-ph/9607141

25. T. Vachaspati, Magnetic fields from cosmological phase transitions. Phys. Lett. B 265, 258261 (1991). https://doi.org/10.1016/ 0370-2693(91)90051-Q

26. K. Enqvist, P. Olesen, On primordial magnetic fields of electroweak origin. Phys. Lett. B 319, 178 (1993). arXiv:hep-ph/9308270

27. M. Joyce, M. Shaposhnikov, Primordial magnetic fields, righthanded electrons, and the Abelian anomaly. Phys. Rev. Lett. 79, 1193 (1997). arXiv:astro-ph/9703005

28. J.M. Cornwall, Speculations on primordial magnetic helicity. Phys. Rev. D 56, 6146 (1997). arXiv:hep-th/9704022

29. S.M. Carroll, G.B. Field, R. Jackiw, Limits on a Lorentz and parity violating modification of electrodynamics. Phys. Rev. D 41, 1231 (1990). https://doi.org/10.1103/PhysRevD.41.1231

30. M.M. Anber, E. Sabancilar, Hypermagnetic fields and baryon asymmetry from pseudoscalar inflation. Phys. Rev. D 92(10), 101501 (2015). arXiv:1507.00744

31. V.B. Semikoz, A.Yu. Smirnov, D.D. Sokoloff, Generation of hypermagnetic helicity and leptogenesis in the early Universe. Phys. Rev. D 93, 103003 (2016). arXiv:1604.02273 [hep-ph]

32. A.J. Long, E. Sabancilar, T. Vachaspati, Leptogenesis and primordial magnetic fields. J. Cosmol. Astropart. Phys. 02, 036 (2014). arXiv:1309.2315 [astro-ph.CO]

33. M. Giovannini, M.E. Shaposhnikov, Primordial hypermagnetic fields and triangle anomaly. Phys. Rev. D 57, 2186 (1998). arXiv:hep-ph/9710234

34. M. Giovannini, Primordial hypermagnetic knots. Phys. Rev. D 61, 063004 (2000). arXiv:hep-ph/9905358

35. K. Kamada, A.J. Long, Baryogenesis from decaying magnetic helicity. Phys. Rev. D 94, 123509 (2016). arXiv:1606.08891 [astroph.CO]

36. K. Bamba, C. Q. Geng, S. H. Ho, Hypermagnetic Baryogenesis. Phys. Lett. B 664, 154-156 (2008). arXiv:0712.1523 [hep-ph]

37. B. Fields, S. Sarkar, Big-Bang nucleosynthesis (2006 Particle Data Group mini-review). J. Phys. G 33, 1 (2006). arXiv:astro-ph/0601514

38. G. Steigman, Primordial nucleosynthesis: the predicted and observed abundances and their consequences. PoS NICXI, 001 (2010). arXiv:1008.4765 [astro-ph.CO]

39. V. Simha, G. Steigman, Constraining the early-Universe baryon density and expansion rate. JCAP 0806, 016 (2008). arXiv:0803.3465 [astro-ph]

40. A.D. Sakharov, Violation of CP invariance, C asymmetry, and baryon asymmetry of the Universe. Pisma Zh. Eksp. Teor. Fiz. 5, $32(1967)$

41. A.D. Sakharov, Violation of CP invariance, C asymmetry, and baryon asymmetry of the Universe. JETP Lett. 5, 24 (1967)

42. A.D. Sakharov, Violation of CP invariance, C asymmetry, and baryon asymmetry of the Universe. Sov. Phys. Usp. 34, 392 (1991)

43. A.D. Sakharov, Violation of CP invariance, C asymmetry, and baryon asymmetry of the Universe. Usp. Fiz. Nauk 161, 61 (1991). https://doi.org/10.1070/PU1991v034n05ABEH002497

44. J.I. Kapusta, B. Muller, M. Stephanov, Relativistic theory of hydrodynamic fluctuations with applications to heavy ion colli- 
sions. Phys. Rev. C 85, 054906 (2012). https://doi.org/10.1103/ PhysRevC.85.054906. arXiv:1112.6405 [nucl-th]

45. S. Jeon, U. Heinz, Introduction to hydrodynamics. Int. J. Mod. Phys. E 24(10), 1530010 (2015). https://doi.org/10.1142/ S0218301315300106. arXiv:1503.03931 [hep-ph]

46. J. de Zarate, J. Sengers, Hydrodynamic Fluctuations in Fluids and Fluid Mixtures (Elsevier Science, Amsterdam, 2006)

47. T. Bhattacharyya, P. Garg, R. Sahoo, P. Samantray, Time evolution of temperature fluctuation in a non-equilibrated system. Eur. Phys. J. A 52(9), 283 (2016). https://doi.org/10.1140/epja/ i2016-16283-x. arXiv:1510.03154 [hep-ph]

48. M.N. Chernodub, Chiral heat wave and mixing of magnetic, vortical and heat waves in chiral media. JHEP 01, 100 (2016). https:// doi.org/10.1007/JHEP01(2016)100. arXiv:1509.01245 [hep-th]

49. Y. Neiman, Y. Oz, Relativistic hydrodynamics with general anomalous charges. JHEP 03, 023 (2011). https://doi.org/10.1007/ JHEP03(2011)023. arXiv:1011.5107 [hep-th]

50. K. Landsteiner, E. Megias, F. Pena-Benitez, Anomalous transport from Kubo formulae. Lect. Notes Phys. 871, 433-468 (2013). https://doi.org/10.1007/978-3-642-37305-3-17. arXiv:1207.5808 [hep-th]

51. N. Yamamoto, Chiral transport of neutrinos in supernovae: neutrino-induced fluid helicity and helical plasma instability. Phys. Rev. D 93(6), 065017 (2016). https://doi.org/10.1103/PhysRevD. 93.065017. arXiv:1511.00933 [astro-ph.HE]

52. K. Landsteiner, Notes on anomaly induced transport. Acta Phys. Pol. B 47, 2617 (2016). https://doi.org/10.5506/APhysPolB.47. 2617. arXiv:1610.04413 [hep-th]

53. S. Anand, J.R. Bhatt, A.K. Pandey, Chiral battery, scaling laws and magnetic fields. JCAP 07, 051 (2017). https://doi.org/10.1088/ 1475-7516/2017/07/051. arXiv:1705.03683 [astro-ph.CO]

54. C.P. Dettmann, N.E. Frankel, V. Kowalenko, Plasma electrodynamics in the expanding Universe. Phys. Rev. D 48, 12 (1993). https:// doi.org/10.1103/PhysRevD.48.5655

55. K. Subramanian, J.D. Barrow, Magnetohydrodynamics in the early Universe and the damping of non-linear Alfven waves. Phys. Rev. D 58, 083502 (1998). arXiv:astro-ph/9712083

56. R. Durrer, A. Neronov, Cosmological magnetic fields: their generation, evolution and observation. Astron. Astrophys. Rev. 21, 62 (2013). arXiv:1303.7121 [astro-ph.CO]

57. J.M. Cline, K. Kainulainen, K.A. Olive, Erasure and regeneration of the primordial baryon asymmetry by sphalerons. Phys. Rev. Lett. 71, 2372 (1993). arXiv:hep-ph/9304321

58. J. M. Cline, K. Kainulainen, K. A. Olive, Protecting the primordial baryon asymmetry from erasure by sphalerons. Phys. Rev. D 49, 6394-6409 (1994). arXiv:hep-ph/9401208 [hep-ph]
59. M. Dvornikov, V.B. Semikoz, Leptogenesis via hypermagnetic fields and baryon asymmetry. J. Cosmol. Astropart. Phys. 1202, 040 (2012). arXiv:1111.6876 [hep-ph] [Erratum: JCAP 1208, E01 (2012)]

60. P. Pavlotic, N. Leite, G. Sigl, Chiral magnetohydrodynamic turbulence. Phys. Rev. D 96, 023504 (2017). arXiv:1612.07382 [astroph.CO]

61. V. Rubakov, A. Tavkhelidze, Stable anomalous states of superdense matter in gauge theories. Phys. Lett. B 165, 109 (1985). https://doi. org/10.1016/0370-2693(85)90701-4

62. V. Rubakov, On the electroweak theory at high fermion density. Prog. Theor. Phys. 75, 366 (1986). https://doi.org/10.1143/PTP. 75.366

63. M. Giovannini, Spectrum of anomalous magnetohydrodynamics. Phys. Rev. D 93, 103518 (2016). arXiv:1509.02126 [hep-th]

64. P. Pavlovic, N. Leite, G. Sigl, Chiral magnetohydrodynamic turbulence. Phys. Rev. D 96, 023504 (2017). arXiv:1612.07382 [astroph.CO]

65. S. Weinberg, Gravitation and Cosmology (Wiley, New York, 1972)

66. R. Banerjee, K. jedamzik, The evolution of cosmic magnetic fields: from the very early universe, to recombination, to the present. Phys. Rev. D 70, 123003 (2004). arXiv:astro-ph/0410032

67. M.E. Shaposhnikov, Structure of the high temperature gauge ground state and electroweak production of the baryon asymmetry. Nucl. Phys. B 299, 797 (1988)

68. M. Dvornikov, V.B. Semikoz, Lepton asymmetry growth in the symmetric phase of an electroweak plasma with hypermagnetic fields versus its washing out by sphalerons. Phys. Rev. D 87, 025023 (2013). arXiv:1212.1416 [astro-ph.CO]

69. A. Monnai, Landau and Eckart frames for relativistic fluids in nuclear collisions. Phys. Rev. C 100(1), 014901 (2019). https:// doi.org/10.1103/PhysRevC.100.014901. arXiv:1904.11940 [nuclth]

70. L.D. Landau, E.M. Lifshitz, Fluid Mechanics (Pergamon Press, New York, 1959)

71. C. Eckart, The thermodynamics of irreversible processes. 1. The simple fluid. Phys. Rev. 58, 267-269 (1940). https://doi.org/10. 1103/PhysRev.58.267

72. M.A. Stephanov, H.U. Yee, No-drag frame for anomalous chiral fluid. Phys. Rev. Lett. 116(12), 122302 (2016). https://doi.org/10. 1103/PhysRevLett.116.122302. arXiv:1508.02396 [hep-th]

73. R. Banerjee, Evolution of primordial magnetic fields in the early Universe. PhD thesis, Ludwig-Maximilians-Universität, München (2002) 